OPEN ACCESS

Edited by:

Luregn J. Schlapbach,

The University of Queensland,

Australia

Reviewed by:

Eric Giannoni,

Lausanne University Hospital (CHUV),

Switzerland

Joseph M. Bliss,

Women \& Infants Hospital of Rhode Island, United States

Alison J. Carey,

College of Medicine, Drexel

University, United States

*Correspondence:

Tobias Strunk

tobias.strunk@health.wa.gov.au

Specialty section:

This article was submitted to

Neonatology,

a section of the journa

Frontiers in Pediatrics

Received: 28 June 2018 Accepted: 02 November 2018 Published: 28 November 2018

Citation: Hibbert JE, Currie A and Strunk T

(2018) Sepsis-Induced Immunosuppression in Neonates.

Front. Pediatr. 6:357.

doi: 10.3389/fped.2018.00357

\section{Sepsis-Induced Immunosuppression in Neonates}

\author{
Julie E. Hibbert ${ }^{1}$, Andrew Currie ${ }^{1,2}$ and Tobias Strunk ${ }^{1,3 *}$ \\ ${ }^{1}$ Centre for Neonatal Research and Education, University of Western Australia, Perth, WA, Australia, ${ }^{2}$ School of Veterinary \\ and Life Sciences, Murdoch University, Perth, WA, Australia, ${ }^{3}$ Neonatal Directorate, King Edward Memorial Hospital for \\ Women, Subiaco, WA, Australia
}

Neonates, especially those born preterm, are at increased risk of sepsis and adverse long-term effects associated with infection-related inflammation. Distinct neonatal immune responses and dysregulated inflammation are central to this unique susceptibility. The traditional separation of sepsis into an initial hyper-inflammatory response followed by hypo-inflammation is continually under review with new developments in this area of research. There is evidence to support the association of mortality in the early acute phase of sepsis with an overwhelming hyper-inflammatory immune response. Emerging evidence from adults suggests that hypo-and hyper-inflammation can occur during any phase of sepsis and that sepsis-immunosuppression is associated with increased mortality, morbidity, and risk to subsequent infection. In adults, sepsis-induced immunosuppression (SII) is characterised by alterations of innate and adaptive immune responses, including, but not limited to, a prominent bias toward anti-inflammatory cytokine secretion, diminished antigen presentation to $T$ cells, and reduced activation and proliferation of $T$ cells. It is unclear if sepsis-immunosuppression also plays a role in the adverse outcomes associated with neonatal sepsis. This review will focus on exploring if key characteristics associated with SII in adults are observed in neonates with sepsis.

Keywords: neonates, preterm infant, innate immunity, adaptive immunity, immune cell function, sepsis, infection, immunosuppression

\section{INFLAMMATION AND SEPSIS-A NEW PARADIGM?}

Sepsis, defined as life-threatening organ dysfunction caused by a dysregulated host response to infection, represents an enormous burden affecting more than 30 million people with potentially 6 million associated deaths per year (1). Until recently, adult sepsis complicated by organ dysfunction, was termed severe sepsis, but is now represented by an increase in the Sequential Organ Failure Assessment (SOFA) score, secondary to the infection cause (2). The SOFA score, based on respiratory, cardiovascular, hepatic, coagulation, renal, and neurological systems, determines the extent of organ function and an increase of two points or more is associated with in-hospital mortality of $>10 \%$ (2). Septic shock is defined as sepsis with circulatory and cellular/metabolic abnormalities that substantially increase mortality (2). Recent evidence from critically ill adults with sepsis and septic shock suggests the extent of recovery from sepsis may depend on the host's ability to orchestrate both the pro-inflammatory and hypo-inflammatory responses to achieve immunological homoeostasis following infection $(3,4)$. Hotchkiss et al., described three potential inflammatory responses to sepsis, and acknowledged that the immune 
response to sepsis is determined by many factors, including pathogen virulence and comorbidities (4). Firstly, at the onset of sepsis the pro-inflammatory response can dominate, even though both the pro-inflammatory and anti-inflammatory responses are initiated, and lead to an overwhelming hyper-inflammatory state that may cause multiple organ dysfunction and death within 1-2 days. Secondly, in patients with impaired immune responses due to comorbidities, the hyper-inflammatory phase may be absent or reduced and a profound anti-inflammatory state may occur, which may lead to further impaired immunity with increased risk of nosocomial infections and higher risk of death 10-14 days following sepsis onset. Thirdly, the immune response cycles between hyper-inflammatory and hypo-inflammatory states and death can occur in either state. With this response, there is an increased probability of the patient developing overwhelming immunosuppression as the infection persists (4).

There is increasing evidence to support the role of immunosuppression in sepsis (4). Critically ill adults with sepsis and septic shock may develop sepsis-induced immunosuppression (SII), a phenomenon of persistent systemic hypo-inflammation that compromises many immune functions, prevents bacterial elimination and immune homeostasis (3-7). Importantly, SII is associated with increased risk of multi-organ failure and mortality, and ongoing immunosuppression results in prolonged (10-14 days) susceptibility to secondary viral and bacterial infections $(3-6,8)$.

Immunologically, SII in adults is incompletely characterised, but is commonly associated with altered functions of the complex network of innate and adaptive immune responses to infection. Specifically, neutrophils, monocytes, macrophages, and dendritic cells (DCs) display a prominent shift of phenotype and function toward an impaired inflammatory response. This further includes decreased bactericidal defences in neutrophils (including oxidative burst and low intracellular expression of myeloperoxidase and lactoferrin), reduced neutrophil chemotaxis, biased anti-inflammatory cytokine secretion with an increased interleukin (IL)-10 to tumour necrosis factor alpha $(\mathrm{TNF} \alpha)$ ratio. There is also reduced expression of the major histocompatibility complex (MHC) II cell surface receptor human leukocyte antigen-DR (HLADR) and consequently impaired antigen-presenting capacity of DCs and monocytes $(3,4,6,7)$. Changes to the adaptive immune response in adults with SII include: reduced activation of $\mathrm{T}$ cells through diminished cell surface expression of the co-stimulatory molecules CD80 and CD86 on antigen presenting cells (APCs); inhibition of $\mathrm{T}$ cell proliferation due to expansion of cell populations with immunosuppressive function, such as immature neutrophils, myeloid-derived suppressor cells (MDSCs), and regulatory $\mathrm{T}$ cells (Tregs); reduced effector functions of $\mathrm{T}$ cells, $\mathrm{B}$ cells, and natural killer cells; and $\mathrm{T}$ cell exhaustion, typified by decreased $\mathrm{T}$ cell activation, reduced ability to produce cytokines, and decreased cytotoxic functions $(3,4,6,7)$. Sepsis-induced apoptosis of DCs, CD4+ and CD8+ T cells and B cells occurs in primary immune organs such as blood, bone marrow, spleen, and thymus, resulting in an overwhelming depletion of immune cells $(3,4,6,7)$.
The suppressive effect of endotoxin tolerance, induced by repeat or long-term exposure to bacterial endotoxins, like lipopolysaccharide (LPS), mediates immune dysfunction through reprogramming of cell signalling and is associated with immunosuppression observed in the later-stage of adult sepsis ( 7 , 9). Leukocytes from adult patients with sepsis behave similarly to in vitro endotoxin-tolerised cells, with a reduced responsiveness to produce cytokines, especially $\mathrm{TNF} \alpha$, upon re-challenge with LPS (10). The molecular mechanism is unclear, but Pena and colleagues have recently identified an endotoxin tolerance gene signature that may predict sepsis and organ dysfunction in adults with sepsis (9). Murine macrophages challenged with Grampositive bacteria can also induce endotoxin tolerance, termed as cross-tolerance, to a lesser extent than LPS (11), but no associated gene signature has yet been reported.

\section{SEPSIS-INDUCED IMMUNOSUPPRESSION AND THE NEONATE}

Neonatal immune development is complex, incompletely understood and orchestrated by many factors, including intra- and extra-uterine exposure to antigens and commensal organisms (12-16). Immune development in infants born preterm ( $<37$ weeks' gestation) may be further altered by perinatal exposures to corticosteroids and antibiotics and the unique environmental influences associated with prolonged hospital stay (e.g., mechanical ventilation, use of indwelling plastic devices, parenteral nutrition, invasive procedures, and exposure to nosocomial microbes) $(14,15,17-19)$.

Despite advances in neonatal care, sepsis remains a significant cause of morbidity among neonates and is one of the most common causes of neonatal death, accounting for over fourhundred and twenty thousand deaths per year (20). Sepsis is a common complication that affects up to $40 \%$ of neonates born $<28$ weeks' gestation $(21,22)$. Chronic long-term morbidities, such as lung disease and neurodevelopmental impairment, are further increased among infants who acquire nosocomial sepsis $(23,24)$. Inflammation-related brain injury and the associated long-term effects are clearly evident in preterm infants with sepsis (23), and have also been observed in adults with sepsis (25).

The increased risk of sepsis-associated morbidity and mortality in neonates is largely attributed to immature innate immune functions resulting in dysregulated pro-inflammatory responses to systemic infection-often referred to as a "cytokine storm" (26-30). The mortality rate in infants with sepsis is $10-16 \%$, with $50-57 \%$ of neonates die within the first 3 days of sepsis onset, $12-20 \%$ within $4-7$ days and $23-39 \%$ after 7 days $(31,32)$. Similar to adults $(4)$, there is strong evidence to support the association of mortality in the acute phase, i.e., within first days of sepsis, with a dysregulated pro-inflammatory immune response $(28-30,33,34)$. However, emerging evidence suggests that the immature immune response to infection in neonates is characteristically similar to the endotoxin tolerance phenotype observed in critically ill adults with sepsis $(7,35)$. This is evident despite Gram-positive bacteria being the major causative organisms in neonatal sepsis (36), unlike in adult sepsis 
(37). In the neonatal setting, the risk of sepsis may be mediated by a relative inability to initiate appropriate hyper-inflammatory responses which, along with a predominant hypo-inflammatory response, actively causes immunosuppression (35). In keeping with findings in adult SII, Gervassi and colleagues proposed that the distinct neonatal responses to invasive microbes, as well as to vaccines, may be at least partly explained by active immune suppression, such as inhibition of $\mathrm{T}$ cell proliferation and function by Tregs and MDSCs, and the potential for B cells to skew the immune response toward an anti-inflammatory $\mathrm{T}$ helper 2 response (38).

Sepsis-induced immunosuppression has not yet been defined in neonates. In adults, SII signifies sepsis severity, septic shock, and mortality $(3,4,6,7)$. Identifying immunosuppression in neonates may not be as straight forward for several reasons, namely: the lack of a globally accepted definition for neonatal sepsis, including grading of severity; the distinct patterns of immune development; and the sparse data available on immune function and response to infection at the time of neonatal sepsis.

Firstly, neonatal sepsis, especially in those born preterm, is not clearly defined. The recently updated Third International Consensus Definitions for sepsis and septic shock in adults are not applicable to children, infants and neonates $(2,39,40)$, and there is no equivalent SOFA score for determining sepsis severity in neonatal sepsis. Further to this, the international paediatric consensus definition for sepsis specifically excludes preterm neonates (39) and performs poorly in term neonates (40). This has widespread implications not only for reporting incidence and prevalence of neonatal sepsis, but for clinical management (accurate diagnosis and appropriate treatment) and the short- and long-term impact on clinical outcomes $(41,42)$. Further to this, the lack of a clear neonatal sepsis definition creates a substantial barrier to identifying predictive markers for sepsis, and improving diagnostic accuracy and speed (41, 43). To date, there is no consensus definition of neonatal sepsis and the current "gold standard" of positive blood culture plus clinical symptoms for the definition of "confirmed sepsis" has significant limitations $(41,44,45)$. To further complicate diagnosis, sepsis can also be classified as "clinical sepsis," with a negative culture in a symptomatic newborn (41).

Secondly, development of immunoregulation is distinct in neonates compared with adults. This includes differences in: (a) absolute numbers of immune cells (e.g., lower neutrophil counts and higher natural killer cell counts in neonates) (12, 46, 47); (b) the proportions of immune cell subtypes (e.g., higher immature/total neutrophil ratio in neonates) $(48,49)$, and (c) levels of various immune plasma proteins (e.g., lower complement, immunoglobulin, antimicrobial peptide levels in neonates) $(12,13)$.

Lastly, there is limited data on neonatal innate immune responses during sepsis, and studies relating immune function to sepsis severity are lacking. Neonatal immune studies commonly utilise cord blood, which is not representative of the immune system at 1-3 weeks of age, when the most common form of neonatal invasive infection, late-onset sepsis (LOS), typically occurs (50). Data available from the time of sepsis can be limited by low number of neonates and confounded by multiple factors, such timing of sample collection, volume of blood sample collection, pathogenesis of the causative organism, time of sepsis onset [e.g., earlyonset sepsis (EOS) $<72 \mathrm{~h}$ after birth and LOS $>72 \mathrm{~h}$ after birth], and sepsis definition (e.g., confirmed vs. clinical). Further to this, time from sepsis onset to death is poorly reported and therefore causative attribution not consistently possible.

Confirming and describing SII in neonates may be instrumental in better defining the immune pathophysiology of neonatal sepsis. This could also aid in the identification of unique biomarkers that could be of clinical utility for immunomonitoring, prediction of outcomes, or even targeted therapeutics. The remainder of this review focusses on characterising immunosuppression in neonatal sepsis and their associated clinical implications. The principal immune functions characterised include cytokine secretion, antigen presentation, expansion of immunosuppressive cells, effector cell function, and sepsis-induced immune cell apoptosis. Information on gestational age (GA), postnatal age at onset of sepsis, classification (i.e., confirmed and clinical sepsis) and age at sepsis-related death in many studies were incomplete and may confound the interpretation. Available data were included in this review and in Tables 1-5 and Supplementary Tables 1, 2. For clarity, when neonatal GA was not described in the publication, we considered any infant born $\geq 37$ weeks' gestation as term and any infant born $<37$ weeks' gestation as preterm.

\section{INFLAMMATORY CYTOKINE SECRETION IN RESPONSE TO INFECTION IN ADULTS AND NEONATES}

\section{Circulating Inflammatory Cytokines in Adult Sepsis}

Together, pro- and anti-inflammatory cytokines influence the innate immune responses to infection (88). Plasma levels of pro-inflammatory cytokines, including TNF $\alpha$, IL-6, IL-8, IL-1 $\beta$, interferon gamma (IFN $\gamma$ ), and the anti-inflammatory cytokines IL-10 and IL-4 are elevated in adults with sepsis and septic shock $(52,56-58,72,89)$-study details described in Table 1. Interestingly, those with septic shock had both higher proand anti-inflammatory levels than patients without shock (57), with the levels of IL-6 and IL-8 positively associated with IL10 levels (56), indicating a correlation with sepsis severity. Further support for the positive association between sepsis severity and immunosuppression are provided by reports of increased mortality in septic patients with elevated IL-10 levels or a high IL-10/TNF $\alpha$ ratio (52, 57-59). Additionally, patients who regained organ function by day 4 following sepsis onset had a significantly higher $\mathrm{TNF} \alpha$ production capacity compared to those with ongoing organ failure by day 6 (51). While there is concomitant secretion of pro- and anti-inflammatory cytokines during sepsis, the increased ratio of IL-10 to TNF $\alpha$ is associated with sepsis mortality and immunosuppression (52, 
TABLE 1 | Sepsis-induced immunosuppression-association of secreted cytokine concentration with sepsis severity in neonates and adults with sepsis.

\begin{tabular}{|c|c|c|c|c|}
\hline Adult or neonatal GA & $\begin{array}{l}\text { Cohort sepsis characteristics ( } n \text { ) } \\
\text { and mortality (if applicable) }\end{array}$ & $\begin{array}{l}\text { Time of blood sampling and age at } \\
\text { sepsis }\end{array}$ & Observation in septic cohort & References \\
\hline \multicolumn{5}{|l|}{ TNF $\alpha$} \\
\hline Adult & $\begin{array}{l}\text { Organ dysfunction during sepsis: } 24 \\
\text { - Organ failure recovery by day } 4: 11 \\
\text { - Organ failure ongoing: } 13\end{array}$ & $\begin{array}{l}\text { Blood samples were taken within } 24 \mathrm{~h} \text { of } \\
\text { initial suspicion of sepsis and on hospital } \\
\text { days } 4 \text { and } 6 \\
\text { Mean (median) age at sepsis } 55 \text { (55) years }\end{array}$ & $\begin{array}{l}\text { Increased TNF } \alpha \text { production } \\
\text { capacity is associated with organ } \\
\text { failure recovery }\end{array}$ & $(51)$ \\
\hline Adult & $\begin{array}{l}\text { Septic shock: } 38 \\
\text { - Survivors: } 22 \\
\text { - Non-survivors: } 16 \\
\text { Mortality within } 28 \text { days after } \\
\text { diagnosis. Time from sepsis onset not } \\
\text { described }\end{array}$ & $\begin{array}{l}\text { Blood samples were taken on days } 1-2 \text {, } \\
3-4,5-7 \text {, and } 8-15 \text { days following } \\
\text { initial suspicion of sepsis } \\
\text { Mean age at sepsis } 64 \text { years }(95 \% \mathrm{Cl} \\
59-69)\end{array}$ & $\begin{array}{l}\text { TNF } \alpha \text { levels were increased in } \\
\text { non-survivors compared to } \\
\text { survivors, but not significantly }\end{array}$ & $(52)$ \\
\hline $\begin{array}{l}\text { Term } \\
\text { (GA range } 37-42 \text { weeks) }\end{array}$ & $\begin{array}{l}\text { Clinical ( } n=10) \text { and confirmed } \\
(n=3) \text { LOS: } 13 \\
\text { - Sepsis: } 4 \\
\text { - Severe sepsis: } 6 \\
\text { - Septic shock: } 3\end{array}$ & $\begin{array}{l}\text { Blood sample was taken at initial } \\
\text { suspicion of sepsis } \\
\text { Median age at sepsis: } 10 \text { days (IQR 7-22 } \\
\text { days) }\end{array}$ & $\begin{array}{l}\text { TNF } \alpha \text { levels were not associated } \\
\text { with sepsis severity }\end{array}$ & (53) \\
\hline $\begin{array}{l}\text { Mix of preterm and } \\
\text { Term } \\
\text { (mean GA not described) }\end{array}$ & $\begin{array}{l}\text { Sepsis: } 50 \\
\text { (EOS: } 41 \text { and LOS: 9) } \\
\text { - Survivors: } 33 \\
\text { - Non-survivors: } 17 \\
\text { Non-sepsis inflammation: } 50 \\
\text { Controls: } 50 \\
\text { Time from sepsis onset to death not } \\
\text { described }\end{array}$ & $\begin{array}{l}\text { Blood samples were taken at sepsis } \\
\text { evaluation (time 0) and on days } 1 \text { and } 2\end{array}$ & $\begin{array}{l}\text { TNF } \alpha \text { was significantly elevated } \\
\text { in non-survivors, compared to } \\
\text { survivors, at time } 0 \text {, but not on } \\
\text { days } 1 \text { or } 2\end{array}$ & $(54)$ \\
\hline $\begin{array}{l}\text { Mix of preterm and } \\
\text { Term } \\
\text { (mean GA } 35.8 \pm 4.1)\end{array}$ & $\begin{array}{l}\text { Confirmed sepsis: } 26 \\
\text { (EOS } n=3 \text { and LOS } n=13 \text { ) } \\
\text { - Survivors: } 17 \\
\text { - Non-survivors: } 9 \\
\text { Controls: } 29 \\
\text { Mortality: } \\
\text { EOS deaths }<2 \text { days: } 5 \text { LOS deaths } \\
\text { >7 days: } 4 \\
\text { Time from sepsis onset to death not } \\
\text { described }\end{array}$ & $\begin{array}{l}\text { Blood samples were taken at sepsis } \\
\text { evaluation before antimicrobial therapy } \\
\text { (time } 0) \text { and on days } 3 \text { and } 7 \\
\text { Mean }( \pm \text { SD) age at sepsis: } \\
\text { EOS } 1.9( \pm 1.1) \text { days } \\
\text { LOS } 20.6( \pm 8.4) \text { days }\end{array}$ & $\begin{array}{l}\text { TNF } \alpha \text { significantly increased } \\
\text { progressively during sepsis in the } \\
\text { non-survivors } \\
\text { TNF } \alpha \text { significantly decreased } \\
\text { progressively during sepsis in the } \\
\text { survivors }\end{array}$ & (55) \\
\hline \multicolumn{5}{|l|}{ IL-6 } \\
\hline Adult & $\begin{array}{l}\text { Septic shock: } 20 \\
\text { SIRS: } 11 \\
\text { Healthy controls: } 10\end{array}$ & $\begin{array}{l}\text { Blood sample was taken within } 24 \mathrm{~h} \text { initial } \\
\text { suspicion of sepsis } \\
\text { Age at septic shock: } 68 \text { years }\end{array}$ & $\begin{array}{l}\text { IL-6 levels higher in septic shock } \\
\text { than controls. Increased levels of } \\
\text { IL-6 were positively associated } \\
\text { with IL-10 levels in septic shock, } \\
\text { indicating correlation with sepsis } \\
\text { severity }\end{array}$ & $(56)$ \\
\hline Adult & $\begin{array}{l}\text { Sepsis:32 } \\
\text { - Sepsis: } 19 \\
\text { - Septic shock: } 13 \\
\text { Healthy controls: } 15\end{array}$ & $\begin{array}{l}\text { Blood sample was taken at initial } \\
\text { suspicion of sepsis } \\
\text { Mean age }( \pm S D) \text { at sepsis: } \\
70.8( \pm 12.7) \text { years }\end{array}$ & $\begin{array}{l}\text { Significantly elevated IL-6 levels } \\
\text { in septic patients compared to } \\
\text { controls Significantly } \\
\text { elevated levels in septic shock } \\
\text { compared to sepsis without } \\
\text { shock }\end{array}$ & $(57)$ \\
\hline $\begin{array}{l}\text { Term } \\
\text { (GA range } 37-42 \text { weeks) }\end{array}$ & $\begin{array}{l}\text { Clinical ( } n=10) \text { and confirmed } \\
(n=3) \text { LOS: } 13 \\
\text { - Sepsis: } 4 \\
\text { - Severe sepsis: } 6 \\
\text { - Septic shock: } 3\end{array}$ & $\begin{array}{l}\text { Blood sample was taken within } 24 \mathrm{~h} \text { initial } \\
\text { suspicion of sepsis } \\
\text { Median (IQR) age at sepsis: } \\
10(7-22) \text { days }\end{array}$ & $\begin{array}{l}\text { Increased IL-6 levels are } \\
\text { associated with septic shock }\end{array}$ & (53) \\
\hline $\begin{array}{l}\text { Mix of preterm and } \\
\text { Term } \\
\text { (mean GA } 35.8 \pm 4.1)\end{array}$ & $\begin{array}{l}\text { Confirmed sepsis: } 26 \\
\text { (EOS } n=13 \text { and LOS } n=13 \text { ) } \\
\text { - Survivors: } 17 \\
\text { - Non-survivors: } 9 \\
\text { Controls: } 29 \\
\text { Mortality: } \\
\text { EOS deaths }<2 \text { days: } 5 \\
\text { LOS deaths }>7 \text { days: } 4 \text { Time from } \\
\text { sepsis onset to death not described }\end{array}$ & $\begin{array}{l}\text { Blood samples were taken at sepsis } \\
\text { evaluation before antimicrobial therapy } \\
\text { (time } 0) \text { and on days } 3 \text { and } 7 \text { following } \\
\text { Mean }( \pm \text { SD) age at sepsis: } \\
\text { EOS } 1.9( \pm 1.1) \text { days } \\
\text { LOS } 20.6( \pm 8.4) \text { days }\end{array}$ & $\begin{array}{l}\text { IL-6 significantly increased } \\
\text { progressively during sepsis } \\
\text { episode in the non-survivors } \\
\text { IL-6 significantly decreased } \\
\text { progressively during sepsis } \\
\text { episode in the survivors }\end{array}$ & (55) \\
\hline
\end{tabular}


TABLE 1 | Continued

\begin{tabular}{|c|c|c|c|c|}
\hline Adult or neonatal GA & $\begin{array}{l}\text { Cohort sepsis characteristics }(n) \\
\text { and mortality (if applicable) }\end{array}$ & $\begin{array}{l}\text { Time of blood sampling and age at } \\
\text { sepsis }\end{array}$ & Observation in septic cohort & References \\
\hline $\begin{array}{l}\text { Mix of preterm and } \\
\text { Term } \\
\text { (mean GA not described) }\end{array}$ & $\begin{array}{l}\text { Confirmed sepsis: } 50 \\
\text { (EOS } n=41 \text { and LOS } n=9 \text { ) } \\
\text { - Survivors: } 33 \\
\text { - Non-survivors: } 17 \\
\text { Non-sepsis inflammation: } 50 \\
\text { Controls: } 50 \\
\text { Time from sepsis onset to death not } \\
\text { described }\end{array}$ & $\begin{array}{l}\text { Blood samples were taken at sepsis } \\
\text { evaluation (time } 0 \text { ) and on days } 1 \text { and } 2 \\
\text { following } \\
\text { Age at sepsis not described }\end{array}$ & $\begin{array}{l}\text { IL-6 was significantly elevated in } \\
\text { non-survivors compared to } \\
\text { survivors, at time all three } \\
\text { timepoints }\end{array}$ & (54) \\
\hline \multicolumn{5}{|l|}{ IL-8 } \\
\hline Adult & $\begin{array}{l}\text { Septic shock: } 20 \\
\text { SIRS: } 11 \\
\text { Healthy controls: } 10\end{array}$ & $\begin{array}{l}\text { Blood sample was taken within } 24 \mathrm{~h} \text { initial } \\
\text { suspicion of sepsis } \\
\text { Age at septic shock: } 68 \text { years }\end{array}$ & $\begin{array}{l}\text { IL-8 levels elevated compared to } \\
\text { SIRS and control. Increased } \\
\text { levels of IL-8 are positively } \\
\text { associated with IL-10 levels in } \\
\text { septic shock, indicating } \\
\text { correlation with sepsis severity }\end{array}$ & (56) \\
\hline $\begin{array}{l}\text { Mix of preterm and } \\
\text { Term } \\
\text { (mean GA } 35.8 \pm 4.1 \text { ) }\end{array}$ & $\begin{array}{l}\text { Confirmed sepsis: } 26 \\
\text { (EOS } n=13 \text { and LOS } n=13 \text { ) } \\
\text { - Survivors: } 17 \\
\text { - Non-survivors: } 9 \\
\text { Controls: } 29 \\
\text { - Mortality: } \\
\text { EOS deaths }<2 \text { days: } 5 \text { LOS deaths } \\
>7 \text { days: } 4 \text { Time from sepsis onset to } \\
\text { death not described }\end{array}$ & $\begin{array}{l}\text { Blood samples were taken at sepsis } \\
\text { evaluation before antimicrobial therapy } \\
\text { (time } 0) \text { and on days } 3 \text { and } 7 \\
\text { Mean }( \pm \text { SD) age at: } \\
\text { EOS } 1.9( \pm 1.1) \text { days } \\
\text { LOS } 20.6( \pm 8.4) \text { days }\end{array}$ & $\begin{array}{l}\text { IL-8 increased progressively } \\
\text { during sepsis episode in the } \\
\text { non-survivors (only significantly } \\
\text { between time } 0 \text { and day } 3 \text { ) } \\
\text { IL-8 significantly decreased } \\
\text { progressively during sepsis } \\
\text { episode in the survivors }\end{array}$ & (55) \\
\hline $\begin{array}{l}\text { Mix of preterm and } \\
\text { Term } \\
\text { (mean GA not described) }\end{array}$ & $\begin{array}{l}\text { Sepsis: } 50 \\
\text { (EOS } n=41 \text { and } \operatorname{LOS} n=9 \text { ) } \\
\text { - Survivors: } 33 \\
\text { - Non-survivors: } 17 \\
\text { Non-sepsis inflammation: } 50 \\
\text { Controls: } 50 \\
\text { Time from sepsis onset to death not } \\
\text { described }\end{array}$ & $\begin{array}{l}\text { Blood samples were taken at sepsis } \\
\text { evaluation (time } 0 \text { ) and on days } 1 \text { and } 2 \\
\text { Age at sepsis not described }\end{array}$ & $\begin{array}{l}\text { IL-8 was significantly elevated in } \\
\text { non-survivors compared to } \\
\text { survivors, at time all three } \\
\text { timepoints }\end{array}$ & (54) \\
\hline Adult & $\begin{array}{l}\text { Septic shock: } 38 \\
\text { - Survivors: } 22 \\
\text { - Non-survivors: } 16 \\
\text { Mortality within } 28 \text { days after } \\
\text { diagnosis. Time from sepsis onset to } \\
\text { death not described }\end{array}$ & $\begin{array}{l}\text { Blood samples were taken on days } 1-2 \text {, } \\
3-4,5-7 \text {, and } 8-15 \text { days following initial } \\
\text { suspicion of sepsis } \\
\text { Mean age at sepsis: } \\
64 \text { years ( } 95 \% \text { Cl } 59-69)\end{array}$ & $\begin{array}{l}\text { IL-10 levels were significantly } \\
\text { elevated throughout the septic } \\
\text { episode in non-survivors } \\
\text { compared to survivors }\end{array}$ & (52) \\
\hline Adult & $\begin{array}{l}\text { Infection (includes more than only } \\
\text { sepsis): } 399 \\
\text { - Survivors: } 366 \\
\text { - Non-survivors: } 33 \\
\text { Time from sepsis onset to death } \\
\text { unclear }\end{array}$ & $\begin{array}{l}\text { Blood sample was taken when empirical } \\
\text { antibiotics commenced } \\
\text { Median (IQR) age at sepsis: } 61(45-77) \\
\text { years }\end{array}$ & $\begin{array}{l}\text { IL-10 levels were significantly } \\
\text { higher in the non-survivors. } \\
\text { Increased IL-10 levels were } \\
\text { associated with increased risk of } \\
\text { mortality }\end{array}$ & (58) \\
\hline Adult & $\begin{array}{l}\text { Septic shock: } 20 \\
\text { SIRS: } 11 \\
\text { Healthy controls: } 10\end{array}$ & $\begin{array}{l}\text { Blood sample was taken within } 24 \mathrm{~h} \text { initial } \\
\text { suspicion of sepsis } \\
\text { Age at septic shock: } 68 \text { years }\end{array}$ & $\begin{array}{l}\text { IL-10 levels more elevated than } \\
\text { controls. Increased levels of IL-6 } \\
\text { and IL- } 8 \text { are positively } \\
\text { associated with IL-10 levels in } \\
\text { septic shock, indicating } \\
\text { correlation with sepsis severity }\end{array}$ & (56) \\
\hline Adult & $\begin{array}{l}\text { Sepsis:32 } \\
\text { - Sepsis: } 19 \\
\text { - Septic shock: } 13 \\
\text { Healthy controls: } 15\end{array}$ & $\begin{array}{l}\text { Blood sample was taken at time of initial } \\
\text { suspicion of sepsis } \\
\text { Mean }( \pm S D) \text { age at sepsis: } \\
70.8( \pm 12.7) \text { years }\end{array}$ & $\begin{array}{l}\text { Significantly elevated IL-10 levels } \\
\text { in septic patients compared to } \\
\text { controls. Significantly elevated } \\
\text { levels in septic shock compared } \\
\text { to sepsis without shock }\end{array}$ & (57) \\
\hline
\end{tabular}


TABLE 1 | Continued

\begin{tabular}{|c|c|c|c|c|}
\hline Adult or neonatal GA & $\begin{array}{l}\text { Cohort sepsis characteristics ( } n \text { ) } \\
\text { and mortality (if applicable) }\end{array}$ & $\begin{array}{l}\text { Time of blood sampling and age at } \\
\text { sepsis }\end{array}$ & Observation in septic cohort & References \\
\hline Adult & $\begin{array}{l}\text { Sepsis: } 61 \\
\text { - Survivors: } 41 \\
\text { - Non-survivors: } 20 \\
\text { Time from sepsis onset to death not } \\
\text { described }\end{array}$ & $\begin{array}{l}\text { Blood sample was taken on day of } \\
\text { admission and the next day } \\
\text { Median (IQR) age at sepsis in years: } \\
\text { Survivors } 52.5 \text { (36-61.5) } \\
\text { Non-survivors } 54.5(42.5-62.5)\end{array}$ & $\begin{array}{l}\text { Significantly elevated IL-10 levels } \\
\text { in non-survivors compared to } \\
\text { survivors }\end{array}$ & (59) \\
\hline Adult & $\begin{array}{l}\text { Post-operative sepsis: } 35 \\
\text { - Survivors: } 24 \\
\text { - Non-survivors: } 11 \\
\text { Post-operative non-sepsis controls: } \\
85 \\
\text { Mean time to mortality } 22.3( \pm 6.6) \\
\text { days. Time from sepsis onset to } \\
\text { death not described }\end{array}$ & $\begin{array}{l}\text { Blood sample was taken at time of initial } \\
\text { suspicion of sepsis } \\
\text { Mean }( \pm \text { SEM) age at sepsis: } \\
61( \pm 2) \text { years }\end{array}$ & $\begin{array}{l}\text { Sepsis is associated with } \\
\text { deficient IL-10 production. } \\
\text { Sepsis survival correlated with } \\
\text { recovery of pro-inflammatory } \\
\text { secretion, but not IL-10 }\end{array}$ & $(60)$ \\
\hline $\begin{array}{l}\text { Term } \\
\text { (GA range } 37-42 \text { weeks) }\end{array}$ & $\begin{array}{l}\text { Clinical ( } n=10) \text { and confirmed } \\
(n=3) \text { LOS: } 13 \\
\text { - Sepsis: } 4 \\
\text { - Severe sepsis: } 6 \\
\text { - Septic shock: } 3\end{array}$ & $\begin{array}{l}\text { Blood sample was taken at time of initial } \\
\text { suspicion of sepsis } \\
\text { Median (IQR) age at sepsis: } 10 \text { (7-22) days }\end{array}$ & $\begin{array}{l}\text { Increased IL-10 levels gradually } \\
\text { increased are with sepsis } \\
\text { severity, but not significantly }\end{array}$ & $(53)$ \\
\hline \multicolumn{5}{|l|}{ IL-10/TNF $\alpha$ RATIO } \\
\hline Adult & $\begin{array}{l}\text { Septic shock: } 38 \\
\text { - Survivors: } 22 \\
\text { - Non-survivors: } 16 \\
\text { Mortality within } 28 \text { days after } \\
\text { diagnosis. Time from sepsis onset to } \\
\text { death not described }\end{array}$ & $\begin{array}{l}\text { Blood samples were taken on days } 1-2 \text {, } \\
3-4,5-7 \text {, and } 8-15 \text { days following initial } \\
\text { suspicion of sepsis } \\
\text { Mean age at sepsis: } \\
64 \text { years ( } 95 \% \text { Cl } 59-69)\end{array}$ & $\begin{array}{l}\text { IL-10/TNF } \alpha \text { ratio was significantly } \\
\text { increased during the first days of } \\
\text { sepsis in non-survivors } \\
\text { compared to survivors }\end{array}$ & $(52)$ \\
\hline Adult & $\begin{array}{l}\text { Infection (includes more than only } \\
\text { sepsis): } 399 \\
\text { - Survivors: } 366 \\
\text { - Non-survivors: } 33 \\
\text { Time from sepsis onset to death } \\
\text { unclear }\end{array}$ & $\begin{array}{l}\text { Blood sample was taken when empirical } \\
\text { antibiotics commenced } \\
\text { Median (IQR) age at sepsis: } 61 \text { (45-77) } \\
\text { years }\end{array}$ & $\begin{array}{l}\text { IL-10/TNF } \alpha \text { ratio was significantly } \\
\text { higher in non-survivors } \\
\text { compared to survivors }\end{array}$ & $(58)$ \\
\hline Neonate of any GA & Not assessed & - & - & - \\
\hline
\end{tabular}

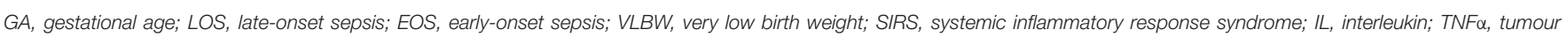
necrosis factor alpha; IFN , type II interferon; IQR, interquartile range; SD, standard deviation; CI, confidence interval.

58), however, the mechanism for this association has yet to be elucidated.

\section{Functional Assessment of Cytokine Secretion in Adult Sepsis}

Pro-inflammatory (TNF $\alpha$, IL-1 $\beta$, IL-6, IL-12) and antiinflammatory (IL-10) responses occur concomitantly in stimulated whole blood and isolated monocytes from septic adults, albeit at a reduced capacity compared to healthy adults $(60,84,89-91)$. Interestingly survival in these patients was associated with the recovery of pro-inflammatory cytokine production, but not IL-10 production-the IL-10/TNF $\alpha$ ratio was not reported (60). A similar pattern of decreased $\mathrm{TNF} \alpha$, IL-6, IFN $\gamma$, and IL-10 was also observed in a post-mortem study of stimulated splenocytes from patients who died of sepsis (84). These results suggest patients with sepsis have a sub-optimal capacity to produce pro- and anti-inflammatory cytokines, which is inversely associated with sepsis severity, especially when IL-10 levels remain relatively higher, eventually leading to organ failure, septic shock, and/or death.

\section{Circulating Inflammatory Cytokines in Neonatal Sepsis}

In both preterm and term neonates with EOS or LOS, the circulating levels of pro-inflammatory cytokines, IL-6 $(28,53-$ $55,92-96)$, IL-8 (53-55), and IFN $\gamma(92,94,95)$ are consistently elevated compared to non-septic neonates. Whereas, TNF $\alpha$ and IL- $1 \beta$ levels are more variable $(28,53,95,96)$ or increased $(28,53-$ $55,92,94,96)$. The inconsistent reports of TNF $\alpha$ and IL- $1 \beta$ concentrations in neonatal sepsis may be a confounded by the kinetics and short half-life of circulating TNF $\alpha$ and IL-1 $\beta$ and the timing of sample collection relative to the onset of sepsis (97, 98). Circulating anti-inflammatory IL-10 concentrations $(28,53,92,94,95)$ are elevated in preterm and term neonates with EOS or LOS compared to non-septic neonates, whereas the concentration of IL-4 is more variable $(28,92,94,95)$. These studies did not report the ratio of IL-10 to TNF $\alpha$. Neonatal and sepsis characteristics, and relevant outcomes of these studies not in relation to sepsis severity are described in the Supplementary Table 1. 
TABLE 2 | Sepsis-induced immunosuppression-association of monocyte surface HLA-DR expression with sepsis severity in neonates and adults with sepsis.

\begin{tabular}{|c|c|c|c|c|}
\hline Adult or neonatal GA & $\begin{array}{l}\text { Cohort sepsis characteristics ( } n \text { ) } \\
\text { and mortality (if applicable) }\end{array}$ & $\begin{array}{l}\text { Time of blood sampling and age at } \\
\text { sepsis }\end{array}$ & Observation in septic cohort & References \\
\hline Adult & $\begin{array}{l}\text { Septic shock: } 38 \\
\text { - Survivors: } 22 \\
\text { - Non-survivors: } 16 \\
\text { Mortality within } 28 \text { days after } \\
\text { diagnosis. Time from sepsis onset to } \\
\text { death not described }\end{array}$ & $\begin{array}{l}\text { Blood samples were taken on days } 1-2 \text {, } \\
3-4,5-7 \text {, and } 8-15 \text { days following initial } \\
\text { suspicion of sepsis } \\
\text { Mean age at sepsis: } \\
64 \text { years (95\% Cl 59-69) }\end{array}$ & $\begin{array}{l}\text { Decreased \% HLA-DR expression } \\
\text { in septic shock } \\
\text { Significantly lower \% HLA-DR } \\
\text { expression in non-survivors } \\
\text { compared to survivors }\end{array}$ & $(52)$ \\
\hline Adult & $\begin{array}{l}\text { Sepsis: } 61 \\
\text { - Survivors: } 41 \\
\text { - Non-survivors: } 20 \\
\text { Time from sepsis onset to death not } \\
\text { described }\end{array}$ & $\begin{array}{l}\text { Blood sample was taken on day of } \\
\text { admission and the next day } \\
\text { Median (IQR) age at sepsis in years: } \\
\text { Survivors } 52.5(36-61.5) \\
\text { Non-survivors } 54.5(42.5-62.5)\end{array}$ & $\begin{array}{l}\text { Decreased HLA-DR expression in } \\
\text { sepsis. Significantly lower in } \\
\text { non-survivors compared to } \\
\text { survivors }\end{array}$ & (59) \\
\hline Adult & $\begin{array}{l}\text { Organ dysfunction during sepsis: } 37 \\
\text { SIRS: } 13 \\
\text { Healthy control: } 20\end{array}$ & $\begin{array}{l}\text { Blood sample was taken within } 24 \mathrm{~h} \text { of } \\
\text { sepsis development } \\
\text { Median (IQR) age at sepsis: } \\
69.4( \pm 2.7) \text { years }\end{array}$ & $\begin{array}{l}\text { Progressive significant decrease in } \\
\text { CD14/HLA-DR expression in the } \\
\text { organ dysfunction during sepsis } \\
\text { group }\end{array}$ & $(61)$ \\
\hline Adult & $\begin{array}{l}\text { Sepsis/septic shock: } 20 \\
\text { Post-surgical inflammation: } 20 \\
\text { Non-sepsis controls: } 10\end{array}$ & $\begin{array}{l}\text { Blood sample was taken within } 24 \mathrm{~h} \text { of } \\
\text { study inclusion } \\
\text { Median (IQR) age at sepsis: } \\
60 \text { (53-67) years }\end{array}$ & $\begin{array}{l}\text { Decreased HLA-DR surface protein } \\
\text { and mRNA expression in } \\
\text { sepsis/septic shock } \\
\text { TNF } \alpha \text { :HLA-DR ratio correlates } \\
\text { negatively with SOFA score }\end{array}$ & $(62)$ \\
\hline Adult & $\begin{array}{l}\text { Sepsis: } 17 \\
\text { - Survivor: } 6 \\
\text { - Non-survivors: } 11 \\
\text { Non-sepsis controls: } 10 \\
\text { Healthy control: } 12 \\
\text { Time to mortality: } \\
\text { During } 1^{\text {st }} \text { septic episode } n=9 \\
\text { During } 2^{\text {nd }} \text { septic episode } n=2 \\
\text { Time from sepsis onset to death not } \\
\text { described }\end{array}$ & $\begin{array}{l}\text { Blood sample was taken upon admission } \\
\text { to the study } \\
\text { Mean }( \pm \text { SEM) age at sepsis: } \\
71( \pm 5) \text { years }\end{array}$ & $\begin{array}{l}\text { HLA-DR expression significantly } \\
\text { decreased in sepsis group. HLA-DR } \\
\text { expression was significantly lower in } \\
\text { non-survivors, compared to } \\
\text { survivors } \\
6 \text { of } 17 \text { with sepsis later developed } \\
\text { nosocomial infections }\end{array}$ & (63) \\
\hline $\begin{array}{l}\text { Mix of preterm and } \\
\text { Term } \\
\text { (mean GA } 37.5 \pm 3.8 \text { ) }\end{array}$ & $\begin{array}{l}\text { Clinical ( } n=22) \text { and confirmed } \\
\text { ( } n=18) \text { LOS: } 40 \\
\text { - Survivor: } 32 \\
\text { - Non-survivor: } 8 \\
\text { Non-sepsis disorder: } 24 \\
\text { Controls: } 25 \\
\text { Time to mortality: during hospital stay. } \\
\text { Time from sepsis onset to death not } \\
\text { described }\end{array}$ & $\begin{array}{l}\text { Sample collection time not described } \\
\text { Mean }( \pm S D) \text { age at sepsis: } \\
16.3( \pm 5.8) \text { days }\end{array}$ & $\begin{array}{l}\text { Significantly lower HLA-DR } \\
\text { expression in sepsis group } \\
\text { HLA-DR expression was } \\
\text { significantly lower in non-survivors } \\
\text { compared to survivors No } \\
\text { significant difference HLA-DR } \\
\text { expression between term and } \\
\text { preterm } \\
\text { No significant difference HLA-DR } \\
\text { expression between clinical and } \\
\text { confirmed LOS }\end{array}$ & (64) \\
\hline $\begin{array}{l}\text { Mix of moderate preterm } \\
\text { and term (median GA 36; } \\
\text { IQR 32-39 wks) }\end{array}$ & $\begin{array}{l}\text { Clinical ( } n=42) \text { and confirmed } \\
(n=21) \text { EOS and LOS: } 63 \\
\text {-Survivor: } 50 \\
\text {-Non-survivor: } 13 \\
\text { Non-sepsis: } 37 \\
\text { Controls: } 29 \\
\text { Mortality }<30 \text { days } n=13 \text { Time from } \\
\text { sepsis onset to death not described }\end{array}$ & $\begin{array}{l}\text { Blood sample taken upon initial suspicion } \\
\text { of sepsis } \\
\text { Median (IQR) age at sepsis: } \\
4(2-11) \text { days }\end{array}$ & $\begin{array}{l}\text { HLA-DR expression was } \\
\text { significantly decreased in the sepsis } \\
\text { group. Lower, but not significantly, } \\
\text { in non-survivors compared to } \\
\text { survivors }\end{array}$ & (65) \\
\hline $\begin{array}{l}\text { Preterm (mean GA } 31 \pm \\
2 \text { weeks) }\end{array}$ & $\begin{array}{l}\text { EOS: } 22 \\
\text { - Mild sepsis: not described } \\
\text {-Severe sepsis: not described } \\
\text { Controls: Not described }\end{array}$ & $\begin{array}{l}\text { Blood samples taken at admission to } \\
\text { NICU during first } 48 \mathrm{~h} \text { of life, during } \\
\text { infection, and recovery } \\
\text { Mean age at sepsis: Not described }\end{array}$ & $\begin{array}{l}\text { Percent of HLA-DR positive } \\
\text { monocytes significantly recovered in } \\
\text { those with mild sepsis. Percent } \\
\text { expression of HLA-DR on } \\
\text { monocytes significantly dropped } \\
\text { followed by a significant recovery in } \\
\text { those with severe sepsis }\end{array}$ & $(66)^{\star}$ \\
\hline
\end{tabular}

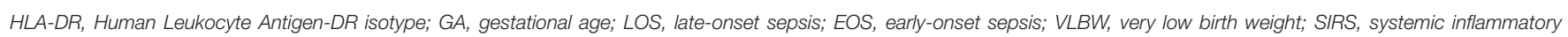
response syndrome; $S D$, standard deviation; IQR, inter-quartile range. *Conference abstract only, limited data available. 
TABLE 3 | Sepsis-induced immunosuppression-association of immunosuppressive cell expansion with sepsis severity in neonates and adults with sepsis.

\begin{tabular}{|c|c|c|c|c|}
\hline Adult or neonatal GA & $\begin{array}{l}\text { Cohort sepsis characteristics }(n) \\
\text { and mortality (if applicable) }\end{array}$ & $\begin{array}{l}\text { Time of blood sampling and age } \\
\text { at sepsis }\end{array}$ & Observation in septic cohort & References \\
\hline \multicolumn{5}{|c|}{ IMMATURE NEUTROPHILS } \\
\hline Adult & $\begin{array}{l}\text { Sepsis: } 83 \\
\text { - Confirmed sepsis: } 51 \\
\text { - Clinical sepsis: } 32 \\
\text { Non-infection SIRS: } 39 \\
\text { Non-SIRS: } 14 \\
\text { Healthy control: } 20\end{array}$ & $\begin{array}{l}\text { Blood sample was taken within } 48 \mathrm{~h} \\
\text { of admission to the intensive care unit } \\
\text { Mean }( \pm \mathrm{SD}) \text { age at sepsis: } \\
\text { Confirmed sepsis: } 62( \pm 16) \text { years } \\
\text { Clinical sepsis: } 66( \pm 13) \text { years }\end{array}$ & $\begin{array}{l}\text { Immature neutrophils were elevated in } \\
\text { the sepsis group. Immature } \\
\text { neutrophils frequency was } \\
\text { significantly higher in confirmed } \\
\text { sepsis compared to clinical sepsis } \\
\text { and non-infection inflammation }\end{array}$ & (68) \\
\hline $\begin{array}{l}\text { Mix of: } \\
\text { Preterm } \\
\leq 28 \text { weeks } \mathrm{GA}(n=21) \\
\text { Preterm } \\
>28-36 \text { weeks GA } \\
(n=123) \\
\text { Term } \\
>36 \text { weeks GA } \\
(n=141)\end{array}$ & $\begin{array}{l}\text { Clinical and confirmed EOS }(n=76) \\
\text { and LOS }(n=134): 210 \\
\text { - Survivor: } 222 \\
\text { - Non-survivor: } 63 \\
\text { No sepsis: } 75 \\
\text { Time from sepsis onset to death not } \\
\text { described }\end{array}$ & $\begin{array}{l}\text { Blood sample was taken upon initial } \\
\text { suspicion of sepsis } \\
\text { Mean }( \pm S D) \text { age at sepsis: } \\
6.7( \pm 7.4) \text { days }\end{array}$ & $\begin{array}{l}\text { Severity of neutrophil left shift } \\
\text { correlates with increased sepsis } \\
\text { mortality risk in both preterm and } \\
\text { term neonates }\end{array}$ & (70) \\
\hline \multicolumn{5}{|c|}{ T REGULATORY CELLS } \\
\hline Adult & $\begin{array}{l}\text { Sepsis: } 80 \\
\text { - Sepsis: } 31 \\
\text { - Organ dysfunction during sepsis: } 33 \\
\text { - Septic shock: } 16 \\
\text { Healthy controls: } 18\end{array}$ & $\begin{array}{l}\text { Blood sample was taken within } 24 \mathrm{~h} \\
\text { after sepsis diagnosis } \\
\text { Median (IQR) age at sepsis: } \\
\text { Sepsis: } 45 \text { (28-72) years } \\
\text { Organ dysfunction during sepsis: } 54 \\
\text { (18-87) years } \\
\text { Septic shock: } 64 \text { (18-84) years }\end{array}$ & $\begin{array}{l}\text { Increased Treg mRNA in sepsis } \\
\text { patients }\end{array}$ & (72) \\
\hline Adult & $\begin{array}{l}\text { Sepsis: } 32 \\
\text { - Sepsis: } 19 \\
\text { - Septic shock: } 13 \\
\text { Healthy controls: } 10\end{array}$ & $\begin{array}{l}\text { Blood sample was taken at time of } \\
\text { sepsis diagnosis } \\
\text { Mean }( \pm \text { SD) age at sepsis: } \\
70.8( \pm 12.7) \text { years }\end{array}$ & $\begin{array}{l}\text { Significantly increased Tregs in CD4 } \\
\text { T cells in sepsis group. Significantly } \\
\text { higher in septic shock than sepsis } \\
\text { without shock }\end{array}$ & (57) \\
\hline Adult & $\begin{array}{l}\text { Septic shock: } 16 \\
\text { - Survivor: } 7 \\
\text { - Non-survivor: } 9 \\
\text { Healthy controls: } 36\end{array}$ & $\begin{array}{l}\text { Blood sampling was taken on days } 1 \text {, } \\
3,5 \text { and } 7-10 \text { following sepsis onset } \\
\text { Mean age at sepsis: } 54 \text { years }\end{array}$ & $\begin{array}{l}\text { Elevated circulating } \mathrm{CD}^{+} \text {Treg cells } \\
\text { in the sepsis group. CD4 }{ }^{+} \text {Treg more } \\
\text { elevated in non-survivors compared } \\
\text { to survivors }\end{array}$ & (73) \\
\hline Adult & $\begin{array}{l}\text { Sepsis: } 118 \\
\text { - Sepsis: } 78 \\
\text { - Septic shock: } 40 \\
\text { Healthy control: } 21\end{array}$ & $\begin{array}{l}\text { Blood sample was taken the day of } \\
\text { study inclusion } \\
\text { Median (IQR) age at: } \\
\text { Sepsis: } 73.5 \text { (62-81) years } \\
\text { Septic shock: } 78.5 \text { (60-84) years }\end{array}$ & $\begin{array}{l}\text { Increased Tregs in } \mathrm{CD}^{+} \mathrm{T} \text { cells in } \\
\text { the sepsis group }\end{array}$ & (74) \\
\hline
\end{tabular}




\begin{tabular}{|c|c|c|c|c|}
\hline Adult or neonatal GA & $\begin{array}{l}\text { Cohort sepsis characteristics }(n) \\
\text { and mortality (if applicable) }\end{array}$ & $\begin{array}{l}\text { Time of blood sampling and age } \\
\text { at sepsis }\end{array}$ & Observation in septic cohort & References \\
\hline Adult & $\begin{array}{l}\text { Sepsis: } 42 \\
\text { - Survivor: } 23 \\
\text { - Non-survivor: } 19 \\
\text { Healthy control: } 14 \\
\text { Time to mortality: <28 days. Time } \\
\text { from sepsis onset to death not } \\
\text { described }\end{array}$ & $\begin{array}{l}\text { Blood samples were taken days } 0 \\
\text { and day } 5 \\
\text { Mean }( \pm S D) \text { age at sepsis: } \\
49.1( \pm 10.2) \text { years }\end{array}$ & $\begin{array}{l}\text { Increased CD39+ Tregs in the sepsis } \\
\text { group. Higher Treg expression in } \\
\text { those with organ failure and } \\
\text { non-survivors }\end{array}$ & $(75)$ \\
\hline Neonate of any GA & Not assessed & - & - & - \\
\hline \multicolumn{5}{|c|}{ MYELOID DERIVED SUPPRESSOR CELLS } \\
\hline Adult & $\begin{array}{l}\text { Sepsis: } 94 \\
\text { - Organ dysfunction during sepsis: } 22 \\
\text { - Septic shock: } 72 \\
\text { Non-septic ICU: } 11 \\
\text { Healthy controls: } 67\end{array}$ & $\begin{array}{l}\text { Blood sample taken within } 3 \text { days of } \\
\text { sepsis diagnosis } \\
\text { Median (IQR) age, in years, at: } \\
\text { Organ dysfunction during sepsis: } 57 \\
(41-75) \\
\text { Septic shock: } 63 \text { (53-73) }\end{array}$ & $\begin{array}{l}\text { In the sepsis group MDSC genes are } \\
\text { up-regulated, G-MDSCs expanded } \\
\text { and plasma MDSC mediator levels } \\
\text { are increased }\end{array}$ & $(76)$ \\
\hline Adult & $\begin{array}{l}\text { Septic shock: } 74 \\
\text { Healthy controls: } 18\end{array}$ & $\begin{array}{l}\text { Blood samples were taken within } 12 \mathrm{~h} \\
\text { of sepsis diagnosis, and on days } 1,4 \text {, } \\
7,14,21 \text { and } 28 \\
\text { Mean age at sepsis: } 60 \text { years }\end{array}$ & $\begin{array}{l}\text { MDSCs persistently increased in the } \\
\text { septic shock group. MDSCs were } \\
\text { functionally immunosuppressive }\end{array}$ & $(77)$ \\
\hline Adult & $\begin{array}{l}\text { Sepsis: } 24 \\
\text { - Sepsis: } 12 \\
\text { - Septic shock: } 12 \\
\text { Non-sepsis: } 12\end{array}$ & $\begin{array}{l}\text { Blood samples were taken at } \\
\text { enrolment, and on days } 2-4 \text { and } \\
\text { 7-discharge } \\
\text { Median (IQR) age at: } \\
\text { Sepsis: } 45 \text { (39-55) years } \\
\text { Septic shock: } 52 \text { (45-57) years }\end{array}$ & $\begin{array}{l}\text { G-MDSCs were increased in the } \\
\text { sepsis group. G-MDSCs were } \\
\text { significantly higher in septic shock } \\
\text { compared to sepsis without shock. } \\
\text { G-MDSCs were functionally } \\
\text { immunosuppressive }\end{array}$ & $(78)$ \\
\hline Neonate of any GA & Not assessed & - & - & - \\
\hline
\end{tabular}

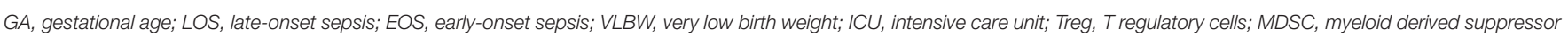
cells; G-MDSC, granulocytic-myeloid derived suppressor cells; SIRS, systemic inflammatory response syndrome; SD, standard deviation; IQR, inter-quartile range.

Gestational age may significantly influence the neonatal cytokine response to infection. In 14 very preterm (mean GA $28.7 \pm 1.3$ weeks) and 12 moderately preterm (mean GA $34.6 \pm 1.8$ weeks) neonates with confirmed or clinical sepsis (including LOS and EOS), the cytokine profiles differed (92). During sepsis, the levels of IFN $\gamma$, IL-6, IL-10, and IL-4 were significantly elevated in the moderate preterm group only. In contrast, the levels of TNF $\alpha$ did not significantly change from pre-sepsis to during sepsis in either group. These results suggest that increasing GA may be associated with a more robust proand anti-inflammatory response. While the lack of inflammatory response in very preterm infants may explain the increased incidence and severity of sepsis (99). The results from this small study do not allow firm conclusions on neonatal clinical outcomes.

Increased IL-6, IL-1 $\beta$, and IL-8 cytokine production might be associated with sepsis severity and/or mortality in neonates with sepsis (53-55)—study details described in Table 1. SilveiraLessa and colleagues investigated cytokine production in 13 term (GA range 37-42 weeks) neonates with confirmed $(n=3)$ and clinical $(n=10)$ LOS, including 6 with severe sepsis [classified as per the international paediatric consensus definition for sepsis (39)] and 3 with septic shock (53). Higher IL-6 and IL-1 $\beta$ levels were significantly associated with septic shock $(n=3)$ and mortality $(n=2)$, respectively (53). Increased levels of IL-8 and IL-10 were associated with sepsis, whereas TNF $\alpha$ was not changed. The sample size in this study was small, thus limiting the interpretation of significant changes in cytokine levels associated with sepsis severity. Similarly, increased levels of IL-6 and IL-8 persisted for longer in preterm and term neonates with fatal LOS and EOS (combined $n=26$ ), whereas the duration of elevated $\mathrm{TNF} \alpha$ levels was variable $(54,55)$. Similar to adults $(100,101)$, it has been suggested that IL-6 concentrations are a strong indicator of sepsis prognosis in neonates $(53,54)$. The results from these studies, summarised in Table 1, suggest increased and persistent levels of pro-inflammatory mediators correlate with greater neonatal sepsis severity. However, evidence as to the association between persistent anti-inflammatory mediator levels and clinical sepsis severity in neonates remains inconclusive.

The focus of the above neonatal studies was to characterise patterns of cytokine production in septic neonates as potential predictive or diagnostic tools or markers of development, and not necessarily to associate cytokine responses to clinical outcomes. From these results, we can acknowledge that neonates are capable of eliciting a cytokine response similar to that of adults in response to infection $(52,56-58,72,89)$. We cannot, however, infer that the pattern of cytokine production associated with SII and sepsis severity in adults, is also present in these neonates.

\section{Functional Assessment of Cytokine Secretion in Neonatal Sepsis}

One study has assessed monocyte cytokine production in 32 extremely (median gestation 25.5 weeks, range $<28$ weeks) and 44 very (median gestation 29 weeks, range $28-32$ weeks) preterm 
TABLE 4 | Sepsis-induced immunosuppression-association of effector cell function and programmed cell death-1 receptor expression with sepsis severity in neonates and adults with sepsis.

\begin{tabular}{|c|c|c|c|c|}
\hline Adult or neonatal GA & $\begin{array}{l}\text { Cohort sepsis characteristics ( } n \text { ) } \\
\text { and mortality (if applicable) }\end{array}$ & $\begin{array}{l}\text { Time of blood sampling and age at } \\
\text { sepsis }\end{array}$ & Observation in septic cohort & References \\
\hline Adult & $\begin{array}{l}\text { Sepsis: } 118 \\
\text { - Sepsis: } 78 \\
\text { - Septic shock: } 40 \\
\text { Healthy control: } 21\end{array}$ & $\begin{array}{l}\text { Blood sample was taken on day of study } \\
\text { inclusion } \\
\text { Median (IQR) age at: } \\
\text { Sepsis: } 73.5 \text { (62-81) years } \\
\text { Septic shock: } 78.5 \text { (60-84) years }\end{array}$ & $\begin{array}{l}\text { Increased PD-1 expression on } \\
\text { Tregs in sepsis group }\end{array}$ & $(74)$ \\
\hline Adult & $\begin{array}{l}\text { Septic shock: } 64 \\
\text { Trauma control: } 13 \\
\text { Healthy control: } 49\end{array}$ & $\begin{array}{l}\text { Blood samples were taken on days } 1-2 \text {, } \\
3-5 \text {, and } 6-10 \text { after diagnosis } \\
\text { Median (IQR) age at septic shock: } 64 \\
\text { (54-73) years }\end{array}$ & $\begin{array}{l}\text { Increased PD-1, PD-L1 } \\
\text { expression on monocytes, and } \\
\mathrm{CD}^{+} \mathrm{T} \text { cells in septic shock } \\
\text { group }\end{array}$ & (79) \\
\hline Adult & $\begin{array}{l}\text { Sepsis: } 135 \\
\text { - Sepsis: } 59 \\
\text { - Septic shock: } 76 \\
\text { Healthy control: } 29\end{array}$ & $\begin{array}{l}\text { Blood samples were taken 3-4 days } \\
\text { after onset of symptoms } \\
\text { Median (IQR) age at: } \\
\text { Sepsis: } 71 \text { (66-78) years } \\
\text { Septic shock: } 71(61-78) \text { years }\end{array}$ & $\begin{array}{l}\text { Increased PD-L1 expression on } \\
\text { monocytes in the sepsis group }\end{array}$ & $(80)$ \\
\hline $\begin{array}{l}\text { VLBW }<1,500 \mathrm{~g} \text { and } \leq 32 \\
\text { weeks GA (mean GA } 26.8 \\
\text { weeks) }\end{array}$ & $\begin{array}{l}\text { LOS: } 39 \\
\text { - Sepsis: } 28 \\
\text { - Septic shock: } 5 \\
\text { Non-survivors: } 6 \\
\text { Control: NA } \\
\text { Time to mortality during } \\
\text { hospitalisation } \\
\text { Time from sepsis onset to death not } \\
\text { described }\end{array}$ & $\begin{array}{l}\text { Blood sample was taken within } 24 \mathrm{~h} \text { of } \\
\text { symptom onset } \\
\text { Age at sepsis not described }\end{array}$ & $\begin{array}{l}\text { Increased PD-L1 expression on } \\
\text { monocytes in sepsis group. } \\
\text { Significant increases in those } \\
\text { with septic shock and/or death } \\
\text { compared to survivors of sepsis } \\
\text { without shock }\end{array}$ & (81) \\
\hline
\end{tabular}

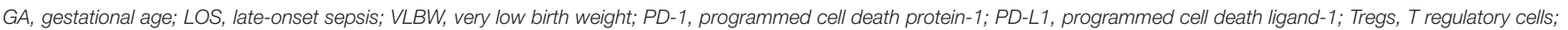
$S D$, standard deviation; IQR, inter-quartile range.

neonates with confirmed $(n=38)$ and clinical $(n=38)$ LOS (102). The authors of this report found that following monocyte stimulation with Pam3Cys, both groups produced equivalent IL- $1 \beta$, but extremely preterm neonates produced higher IL-18 (102). These results highlight the influence GA has on neonatal immune regulation, but outcomes are limited. There is a need to further investigate if immune cell dysfunction at the time of sepsis underpins immunosuppression in neonatal sepsis.

\section{REDUCED MHC CLASS II EXPRESSION IN ADULT AND NEONATAL SEPSIS \\ HLA-DR Expression in Adult Sepsis}

The upregulation of HLA-DR cell surface expression on APCs is a hallmark of APC activation and essential for increased presentation of antigens to naïve $\mathrm{T}$ cells, a critical step for initiating the adaptive immune response (103). Low HLADR expression associated with SII is often referred to as immunoparalysis $(6,104,105)$ and the established cut-off for identifying immunoparalysis in adult patients with sepsis is $<30 \%$ HLA-DR positive monocytes $(6,106,107)$. In adults, sepsis and septic shock have been shown to negatively affect HLA-DR cell surface expression and cause immunosuppression $(52,61-$ $63,73,76,108)$ - study details summarised in Table 2. Low HLADR expression on monocytes and immunoparalysis are related to sepsis severity as shown by a significant increase in SOFA scores in adults with sepsis $(61,62)$. Monocyte HLA-DR expression is also significantly lower in sepsis non-survivors compared to survivors $(52,59,61,63)$.
Low HLA-DR expression on adult monocytes during sepsis is associated with altered immune responses, including imbalanced secretion of pro- and anti-inflammatory mediators and reduced antigen presentation capacity, and importantly sepsis severity and mortality (52, 59, 61-63). Decreased monocyte HLA-DR expression in critically ill adults with sepsis or septic shock has also been associated with a prominent shift toward significantly increased circulating levels of IL-10 (52, 59, 63). Interestingly, IL-10 mediates HLA-DR expression on monocytes (109-111), suggesting that HLA-DR expression could be a marker of SIIrelated cytokine changes.

In a study of 17 critically ill adults with sepsis, decreased expression of HLA-DR and CD86 on monocytes and CD28 on lymphocytes was significantly associated with reduced antigen presentation (63). Although the authors did not find any association between the levels of HLA-DR expression or antigen presentation and development of secondary infections, 6 of the 8 patients who survived sepsis went on to develop a secondary infection, 2 of whom later died (63).

\section{Low HLA-DR Expression in Neonatal Sepsis}

Several studies reported a decrease in monocyte HLA-DR expression in preterm and term neonates with confirmed or clinical sepsis (including EOS and LOS) (64-66, 93, 112). Neonatal and sepsis characteristics, and relevant outcomes of these studies not in relation to sepsis severity are described in the Supplementary Table 2. Decreased HLA-DR expression 
TABLE 5 | Sepsis-induced immunosuppression-association of sepsis-induced immune cell apoptosis and depletion with sepsis severity in neonates and adults with sepsis.

\begin{tabular}{|c|c|c|c|c|}
\hline Adult or neonatal GA & $\begin{array}{l}\text { Cohort sepsis characteristics } \\
\text { (n) and mortality (if applicable) }\end{array}$ & $\begin{array}{l}\text { Time of blood sampling and } \\
\text { age at sepsis }\end{array}$ & Observation in septic cohort & References \\
\hline Adult & $\begin{array}{l}\text { Prospective study: } \\
\text { Sepsis: } 71 \\
\text { Non-sepsis:55 } \\
\text { Healthy control: } 6\end{array}$ & $\begin{array}{l}\text { Blood samples were collected at } \\
\text { various times during sepsis } \\
\text { Mean age range at sepsis: } 57-59\end{array}$ & $\begin{array}{l}\text { Increased T cell, B cell, and } \\
\text { dendritic cell apoptosis in the } \\
\text { sepsis group }\end{array}$ & (82) \\
\hline Adult & $\begin{array}{l}\text { Prospective study: } \\
\text { Septic shock: } 19 \\
\text { Healthy control: } 22\end{array}$ & $\begin{array}{l}\text { Blood sample was collected at } \\
\text { time of study inclusion } \\
\text { Mean }( \pm \text { SD) age at sepsis: } \\
58( \pm 4) \text { years }\end{array}$ & $\begin{array}{l}\text { Marked increase in apoptosis of } \\
C D 4^{+} \text {and CD8 } 8^{+} T \text { cells and B } \\
\text { cells in the septic shock group }\end{array}$ & (83) \\
\hline Adult & $\begin{array}{l}\text { Post-mortem study: } \\
\text { Organ dysfunction during sepsis: } \\
40 \\
\text { Trauma control: } 29 \\
\text { Median (range) days of sepsis: } 4 \\
\text { (1-40). Time from sepsis onset to } \\
\text { death not described }\end{array}$ & $\begin{array}{l}\text { Post-mortem sample collection } \\
\text { occurred } 30-180 \text { min following } \\
\text { death } \\
\text { Mean }( \pm \text { SD) age at organ } \\
\text { dysfunction during sepsis: } 71.7 \\
( \pm 15.9) \text { years }\end{array}$ & $\begin{array}{l}\text { Extensive depletion of splenic } \\
\text { CD4 }{ }^{+} \text {and } C D 8^{+} T \text { cells and } \\
\text { HLA-DR cells in the organ } \\
\text { dysfunction during sepsis group }\end{array}$ & (84) \\
\hline Adult & $\begin{array}{l}\text { Prospective and post-mortem } \\
\text { study Sepsis: } 27 \\
\text { - Survivor: } 2 \\
\text { - Non-survivors: } 25 \\
\text { Non-septic critically ill: } 16 \\
\text { Trauma control: } 25 \\
\text { Mean age of death and time from } \\
\text { sepsis onset to death not } \\
\text { described }\end{array}$ & $\begin{array}{l}\text { Sample collection was either } \\
\text { intraoperatively (survivors) or } \\
\text { post-mortem ( } 15 \text { min to } 6 \mathrm{~h} \\
\text { following death) } \\
\text { Mean age as sepsis not } \\
\text { described }\end{array}$ & $\begin{array}{l}\text { Depletion of splenic CD4+ } \mathrm{T} \\
\text { helper cells and B cells in the } \\
\text { sepsis group }\end{array}$ & (85) \\
\hline $\begin{array}{l}\text { VLBW } \\
<1,500 \mathrm{~g} \\
\text { (approximate mean GA } \\
27 \text { weeks) }\end{array}$ & $\begin{array}{l}\text { EOS: } 5 \\
\text { - Survivor: } 0 \\
\text { - Non-survivors: } 5 \\
\text { LOS: } 15 \\
\text { - Survivor: } 0 \\
\text { - Non-survivors: } 15 \\
\text { Controls: NA Mean }( \pm \text { SD) age of } \\
\text { death: EOS: } 1.6( \pm 0.5) \text { days } \\
\text { LOS:17.8 }( \pm 12.1) \text { days Time from } \\
\text { sepsis onset to death not } \\
\text { described }\end{array}$ & $\begin{array}{l}\text { Post-mortem examination } \\
\text { completed within } 2 \mathrm{~h} \text { of death } \\
\text { Mean }( \pm S D) \text { age at sepsis: } \\
\text { EOS: } 0( \pm 0) \text { days } \\
\text { LOS: } 14.1( \pm 9.9) \text { days }\end{array}$ & $\begin{array}{l}\text { EOS: No cell depletion } \\
\text { LOS: Depletion of thymus } \\
\text { lymphocytes }\end{array}$ & (71) \\
\hline $\begin{array}{l}\text { Moderate preterm } \\
\text { (GA range 35-37 } \\
\text { weeks) }\end{array}$ & $\begin{array}{l}\text { Sepsis: } 6 \\
\text { - Survivor: } 0 \\
\text { - Non-survivor:6 Control mortality: } \\
6 \text { Mean age of death and time } \\
\text { from sepsis onset to death not } \\
\text { described }\end{array}$ & $\begin{array}{l}\text { Post-mortem examination time } \\
\text { not described } \\
\text { Age at sepsis not described }\end{array}$ & $\begin{array}{l}\text { Depletion of neutrophils in the } \\
\text { sepsis group }\end{array}$ & (86) \\
\hline $\begin{array}{l}\text { Mix of preterm and } \\
\text { term } \\
\text { (GA mean } 29.2 \text { (range } \\
\text { 24-38) weeks) }\end{array}$ & $\begin{array}{l}\text { EOS: } 10 \\
\text { - Survivor: } 0 \\
\text { - Non-survivor: } 10 \text { Control } \\
\text { mortality: } 20 \text { Time to mortality } \\
\text { within } 48 \mathrm{~h} \text { after birth. Time from } \\
\text { sepsis onset to death not } \\
\text { described }\end{array}$ & $\begin{array}{l}\text { Post-mortem examination } \\
\text { occurred between } 4 \text { and } 12 \mathrm{~h} \\
\text { following death } \\
\text { Age at sepsis }<48 \mathrm{~h} \text { after birth }\end{array}$ & Depletion of $T$ cells and B cells & (87) \\
\hline
\end{tabular}

GA, gestational age; LOS, late-onset sepsis; EOS, early-onset sepsis; VLBW, very low birth weight; HLA-DR, Human Leukocyte Antigen-DR isotype.

observed in mixed cohorts of preterm and term with neonatal sepsis appears unrelated to the GA $(64,93,112)$. Serial assessment of HLA-DR expression during neonatal sepsis demonstrated that 3 days after sepsis onset, HLA-DR expression in both preterm and term neonates were similar to those without sepsis (93). However, low HLA-DR expression is a possible marker of sepsis-related mortality, as monocyte HLA-DR expression is down-regulated in term and preterm non-survivors of sepsis compared to survivors
(64, 65) - study details summarised in Table 2. In this small study, Genel et al reported a significant decrease in monocyte HLA-DR expression between non-survivor $(n=8)$ and survivor $(n=32)$ preterm and term (median GA 36 weeks) neonates with confirmed $(n=18)$ and clinical $(n=22)$ LOS (mean postnatal age 16.3 days) (64). The preterm and term neonates with $\leq 30 \%$ HLA-DR positive monocytes had a 30 -fold higher risk of mortality (Odds ratio 30 ); with $53.8 \%$ mortality among 
those with $\leq 30 \%$ HLA-DR positive monocytes compared to only $3.7 \%$ in neonates with $>30 \%$ HLA-DR positive monocytes (64), similar to adults with confirmed immunoparalysis (61). Unlike for HLA-DR surface expression levels, the proportion of cells expressing any HLA-DR in neonates is correlated with GA and acts as a predisposing factor for sepsis as reported in 31 very low birth weight infants (VLBW; GA range 23-31 weeks) with clinical $(n=14)$ and confirmed $(n=17)$ sepsis (EOS $n=2$ and LOS $n=29)$ (113). Pradhan et al. suggested that monocyte HLA-DR expression, combined with CD64 expression on neutrophils, may be a useful prognostic marker for neonatal sepsis (65).

There is a decrease in HLA-DR positive monocytes among preterm and term neonates with sepsis, compared to non-septic neonates $(64,66,93,112,114,115)$. Decreased HLA-DR positive monocytes in neonates with sepsis appears unrelated to the GA $(64,93,112)$. Fotopoulos et al. monitored the proportion of HLADR positive monocytes over the course of a septic episode in preterm neonates (mean GA 31 weeks), with and without EOS (66). They reported that the percentage of HLA-DR positive monocytes significantly recovered over the course of sepsis in those neonates with mild sepsis, while those with severe sepsis showed a significant drop followed by a rise only upon recovery (66). While the authors did not provide the criteria for defining sepsis severity, this data may suggest that a decreased percentage of HLA-DR positive monocytes is associated with sepsis severity in neonates, however additional research in this area is essential.

\section{EXPANSION OF IMMUNOSUPPRESSIVE CELLS IN ADULT AND NEONATAL SEPSIS}

\section{Immature Neutrophils in Adult Sepsis}

The ability of immature neutrophils to suppress $\mathrm{T}$ cell proliferation was first observed by Pillay et al. (116), although the mechanism for suppression remains unclear. An increased frequency of immature neutrophils has been observed in adults with sepsis and is associated with sepsis severity, poor clinical outcomes, and increased risk of septic shock and mortality (6769) - study details summarised in Table 3. As sepsis becomes more severe in adults, the increased frequency in immature neutrophil has been shown to be associated with a decrease in T cell proliferation (67).

\section{Immature Neutrophils in Neonatal Sepsis}

While neonates with sepsis have increased numbers of circulating immature neutrophils compared to neonates without sepsis, this is not a reliable diagnostic marker (117-121). There is a paucity of data on whether the $\mathrm{T}$ cell suppressive function of immature neutrophils contributes to sepsis severity, adverse outcomes, and increased mortality in neonatal sepsis. Saied and colleagues evaluated neutrophil left shift for its predictive value in sepsis outcomes in extremely preterm $(n=21$; GA range $\leq 28$ weeks), very/moderate preterm $(n=123$; GA range $>28-36$ weeks), and term $(n=141$; GA range $>36$ weeks $)$ neonates with confirmed or clinical sepsis (EOS $n=76$; LOS $n=134$ ) (70). Although $\mathrm{T}$ cell function was not assessed in this study, they found that an increase in left shift (and hence proportions of immature neutrophils) correlates with increased sepsis mortality risk in preterm and term neonates (70). Further to this, Itoh et al. found a high number of circulating immature neutrophils, in addition to depleted lymphocytes in the thymus and hypertrophic spleen, in $15 \mathrm{VLBW}$ infants $(<1,500 \mathrm{~g})$ that died from confirmed LOS (mean time from sepsis onset to death $3.7 \pm 3.3$ days) (71). Yet, the number of circulating immature neutrophils was only slightly elevated during the initial stage of sepsis the neonates that died from confirmed EOS $(n=5$; mean time from sepsis onset to death $1.6 \pm 0.5$ days) (71). However, the number of neonates with sepsis were low and a non-septic control group was lacking for comparison. The frequency of immature neutrophils and its relation to sepsis severity observed in both neonates and adults are summarised in Table 3. These two studies suggest that immature neutrophils may be associated with worse outcomes with neonatal sepsis, however, further studies with larger sample size and non-septic controls are essential. Whether increased numbers of immature neutrophils are a consequence of sepsis or whether they cause more severe infection due to their immunosuppressive function on $\mathrm{T}$ cells remains to be determined in neonatal sepsis.

\section{Regulatory T Cells (Tregs) in Adult Sepsis}

Tregs play an important role in the maintenance of immune homeostasis, however their role in immunosuppression during sepsis is not entirely clear $(7,122,123)$. Several studies have reported an elevated proportion of Tregs following the onset of sepsis or septic shock in adults, and associated this with an increased risk of immunosuppression, mortality, and morbidity (57, 72-75) - study details summarised in Table 3. The increased risk of mortality associated with sepsis and sepsis shock may be attributed to the immunosuppressive functions of Tregs by: (a) directly inhibiting effector $\mathrm{CD} 4+\mathrm{T}$ cell proliferation and cytokine secretion (124-126); (b) indirectly supressing APC/Tcell receptor mediated $\mathrm{CD} 4+$ and $\mathrm{CD} 8+\mathrm{T}$ cell activation (125-127); (c) suppressing T cell activation through increased expression of programmed cell death-1 (PD-1) receptor (128); or (d) suppressing other immune effector cells such as natural killer cells, B cells, and monocytes (129-131).

\section{Regulatory T Cells (Tregs) in Neonatal Sepsis}

Identifying the role of Tregs in neonatal sepsis is an emerging area of research. The proportion of Tregs is elevated in term neonates with confirmed sepsis $(n=30)$ (132). Likewise, the proportion of Tregs is higher in 22 preterm neonates (mean GA $28.1 \pm 3.7$ weeks) with clinical EOS (133). Sepsis severity and mortality were not discussed in these studies, nor was the age of sepsis onset. Surprisingly, unlike cord blood (134-137), the elevated proportion of Tregs reported in septic neonates may not be affected by GA (133), suggesting sepsis alone, and not gestation, influences Treg frequency, postnatally. There are no data on the potential impact of Tregs on sepsis severity, immunosuppression, or mortality during neonatal sepsis. Treg frequencies and further functional analysis is required to determine whether Tregs suppress $\mathrm{T}$ cell proliferation and function during neonatal sepsis, as observed in adults with SII $(74,122,123,125)$. 


\section{Myeloid-Derived Suppressor Cells (MDSCs) in Adult Sepsis}

In healthy adults, the immature cells of the myeloid lineage, namely monocytic- and granulocytic-MDSCs, rapidly differentiate into DCs, macrophages, and granulocytes and act to preserve innate immunity (138). The expansion of MDSCs to suppress both the innate and adaptive immune responses is a phenomenon under investigation in adults with SII $(77,138)$. MDSC expansion has been observed in adults with sepsis and septic shock $(76-78,138)$, as summarised in Table 3 . The immune suppressive characteristics of MDSC expansion, namely inhibition of $\mathrm{T}$ cell proliferation $(76-78,138)$ and increased secretion of immunosuppressive mediator IL-10 (78, 138), have been observed in adults with sepsis. MDSC expansion is associated with sepsis severity $(77,78)$ and adverse outcomes, such as chronic immune suppression following prolonged MDSC expansion in critically ill adults with sepsis (77). Expansion of MDSCs has also been shown to be associated with higher risk for subsequent nosocomial infections $(76,77)$, a characteristic found among patients with SII. However, the frequency of MDSCs among sepsis survivors and non-survivors was found to be similar, suggesting MDSC expansion alone does not influence mortality (76).

\section{Myeloid-Derived Suppressor Cells (MDSCs) in Neonatal Sepsis}

Only one group has investigated the frequency of MDSCs during neonatal sepsis, and found a significant increase in the frequency of MDSCs in 10 preterm neonates (mean GA $25 \pm 3$ weeks) with early- and late-onset clinical (80\%) and confirmed (20\%) sepsis compared to preterm neonates (GA range 23 to $<37$ weeks) without sepsis (139). No associations with severity or sepsis outcomes were made in this study. With the limited data and small sample size in this publication the characterisation of MDSCs during neonatal sepsis requires further evaluation.

\section{PROLONGED IMMUNOSUPPRESSION AND ALARMINS}

Alarmins (also referred to as damage-associated molecular patterns), such as S100A8 and S100A9, are pro-inflammatory mediators present in low levels in circulating myeloid cells, namely monocytes and granulocytes, even in healthy subjects. S100A8 and S1009A are up-regulated in response to bacterial products, as well as pro- (e.g., TNF $\alpha$ and IL-1 $\beta$ ) and anti-inflammatory cytokines (e.g., IL-10 and transforming growth factor $\beta$ ) (140). Up-regulation of S100A8 and S100A9 positively regulates MDSC frequency and function $(141,142)$, stimulates Treg expansion (140), and induces endotoxin tolerance by rendering phagocytes unresponsive to secondary Toll-Like Receptor-4 stimulation (143). Whether S100A8 and S100A9 function to enhance inflammation or to support immunosuppression is still unclear.

\section{Alarmins in Adult Sepsis}

Plasma levels of S100A8 and S100A9 were elevated, as was upregulation of S100A12, S100A9, and arginase-1 gene expression, in adults with sepsis, compared to non-septic patients in intensive care (76). The increased levels of S100A12, S100A9, and arginase- 1 were associated with MDSC expansion, and high initial levels of granulocytic-MDSCs, arginase-1 and S100A12 were associated with subsequent infections (76). Alarmins, such as the $\mathrm{S} 100$ proteins, may therefore, play multiple roles in adult SII.

\section{Alarmins in Neonatal Sepsis}

S100A8/A9 levels were elevated in eight neonates with confirmed sepsis (144). Gestational age, postnatal age at sepsis onset and relation of alarmin levels to the severity of the sepsis were not discussed. Whether alarmins, such as S100A8/S100A9, play a role in MDSC expansion and immunosuppression in neonatal sepsis requires further investigation.

\section{COMPROMISED T CELL EFFECTOR CELL FUNCTION IN ADULT AND NEONATAL SEPSIS}

\section{Immune Checkpoint Molecule Expression in Adult Sepsis}

The increased expression of the negative co-stimulatory molecule $\mathrm{PD}-1$, and its associated ligand (PD-L1), on circulating monocytes, neutrophils and effector $\mathrm{T}$ cells may contribute to SII (79). Increased expression of PD-1/PD-L1 on monocytes and lymphocytes is associated with decreased monocyte HLADR expression, increased proportions of Tregs, and $\mathrm{T}$ cell exhaustion $(3,4,79,145-147)$. Several groups have reported an over-expression of PD-1 on T cells, including CD4+ and Tregs, and PD-L1 on monocytes in adults with sepsis and septic shock compared to healthy adults $(74,79,80,83,84)$. Increased expression of PD-1 and PD-L1 on lymphocytes and monocytes is associated with more organ dysfunction during sepsis and increased risk of secondary infections and mortality $(74,79$, 80), as summarised in Table 4. In addition, increased PD-L1 expression on monocytes has been shown to be an independent predictor of mortality in septic shock patients (80).

PD-1/PD-1L are inhibitory immune checkpoint molecules and blockade of their function to interact with other immune cells is being explored as a therapeutic agent for reversing the effects of immunosuppression (148). Pre-clinical models of sepsis, have shown that blockade of the PD-1/PD-L1 pathway with an antagonistic anti-PD-L1 antibody improves survival by inhibiting lymphocyte apoptosis and $\mathrm{T}$ cell exhaustion (145, $149,150)$. Further to this, in vitro blockade of the PD-1/PD-L1 pathway in the blood from septic adults, decreases lymphocyte apoptosis, increases pro-inflammatory cytokine production and decreases IL-10 production $(83,146)$. Antibody blockade of PD1 or PD-L1 as an immunomodulatory therapy for reversing immunosuppression is being trialled to improve survival in 
human patients with cancer (151). This promising therapy may spark exploration for PD-1 blockade immunotherapy in sepsis.

\section{Immune Checkpoint Molecule Expression in Neonatal Sepsis}

Despite the interest in exploring PD-1 blockade for reversing immunosuppression in septic adults, there is a paucity of data pertaining to PD-1 and PD-L1 expression or T cell exhaustion in neonates, and importantly neonates with sepsis. PD-1 expression was increased in 34 VLBW $(<1,500 \mathrm{~g}$ and GA range $\leq 32$ weeks) with confirmed LOS, and expression was significantly increased in 5 preterm infants with septic shock (identified using the international paediatric consensus criteria) and/or mortality $(n=6)$ compared to surviving preterm infants without shock (81). The role of GA on PD-1 expression during neonatal sepsis has not been explored. The results from this study, summarised in Table 4, suggest increased PD-1 expression may have an immunosuppressive function in neonatal sepsis, however with the limited available data this interpretation remains inconclusive.

Interestingly, Young and colleagues recently investigated the role of PD-1 in murine neonates and found improved survival in septic PD-1 knockout mice, further supporting the functional importance of PD-1 in neonatal sepsis and related mortality (152). The therapeutic potential for targeted blockade of PD-1 means that this is an area that deserves urgent exploration.

Up-regulation of carcinoembryonic antigen-related celladhesion molecule 1 (CEACAM1), another inhibitory immune checkpoint molecule (153), on $\mathrm{T}$ cells leads to reduced proliferation and cytokine secretion causing $\mathrm{T}$ cell suppression and subsequent prolonged immunosuppression (154, 155). Although not reported in adults with SII, the percentage of CEACAM1-positive CD4+ $\mathrm{T}$ cells in 12 preterm neonates with LOS is increased compared to 16 non-septic controls (155). With the small sample size and limited available data it is inconclusive as to whether increased expression of CEACAM1 on CD4+ T cells contributes to immunosuppression in septic neonates and thus requires further investigation.

\section{SEPSIS-INDUCED IMMUNE CELL APOPTOSIS IN ADULTS AND NEONATES}

\section{Immune Cell Apoptosis in Adult Sepsis}

Cell death is an important step for resolving infection and maintaining immune homeostasis. However, sepsis-induced immune cell apoptosis, resulting in an overwhelming depletion of immune cells, including T cells (CD4+ and CD8+), B cells, and DCs, is evident in prospective studies of adults with sepsis $(82,83)$. Similar findings have been reported in post-mortem studies of adults who died from sepsis, septic shock, and sepsisrelated multiple organ dysfunction $(84,85,156,157)$-study details summarised in Table 5. The degree of immune cell apoptosis has been shown to be correlated with sepsis severity, supporting a role for apoptosis in SII (82). In support of this concept, in mice in vivo prevention of cell death improves sepsis survival (158-162).

\section{Immune Cell Apoptosis in Neonatal Sepsis}

Three post-mortem studies have reported lymphocyte depletion in the spleen, thymus, and bone marrow in both preterm and term neonates that died from EOS and LOS compared to neonates that died of causes other than sepsis $(71,86,87)$. The sample size in all three studies was small, with 5-15 neonates in the sepsis groups. There were conflicting results between the two studies that reported on lymphocyte depletion following EOS $(71,87)$. Only two of the three studies described the time from sepsis onset to death; death occurred within $48 \mathrm{~h}$ after sepsis onset for EOS $(71,87)$ and, on average, $3.7( \pm 3.3)$ days following sepsis onset for LOS (71). These results, summarised in Table 5, suggest both term and preterm neonates with severe sepsis may develop sepsis-induced immune cell apoptosis, however there are no prospective studies to support this conclusion. Sepsisinduced immune cell apoptosis in relation to disease severity has not been assessed in neonates. A mouse model of neonatal sepsis found that blocking necroptosis, programmed cell death triggered by the caspase-independent pathway through death receptors, by inhibition of receptor-interacting protein kinase 1 with necrostatin-1, reduced lung injury associated with sepsis and improved survival (162).

\section{IS SEPSIS-INDUCED IMMUNOSUPPRESSION A FEATURE OF NEONATAL SEPSIS?}

In adults, SII is associated with increased risk of multi-organ failure and mortality as well as susceptibility to secondary viral and bacterial infections $(3,4,6,8)$. Similar clinical characteristics, including increased risk of multi-organ failure and mortality, are observed in neonatal sepsis $(31,163-165)$. It is unclear if these adverse outcomes observed in neonates are due to an overwhelming hyper-inflammatory immune response and/or SII. From the limited data available, it appears that fatal neonatal sepsis may be associated with alterations in immune function that are in agreement with SII findings in adults $(3,4,6)$. The dysregulated immune responses observed in various neonatal studies include imbalanced secretion of proand anti-inflammatory mediators (53-55), diminished HLADR monocyte surface expression (64), expansion of immature neutrophils $(70,71)$, increased expression of PD-1/PD-L1 (81), and depletion of leukocytes $(71,86,87)$. Published neonatal studies are limited by: (i) small sample size of neonates, (ii) incomplete reporting of time from sepsis onset to death, and (iii) the lack of consistent neonatal sepsis definition and objective measures for the degree of severity.

Despite distinct patterns of causative pathogens in adult and neonatal sepsis, similar immune function alterations are observed and endotoxin tolerance appears to be a feature in both adult and neonatal sepsis $(9,35)$. Therefore, SII may occur independently as a feature of the subsequent host response to inflammation.

In adults, SII is associated with increased susceptibility to secondary bacterial infections and associated late mortality (166, 167). Whether this is a result of organ damage or persistent SII 
is unclear. Interestingly, one study reported that the immune alterations associated with SII in adult septic shock survivors continued until discharge from the intensive care unit, but resolved by 6 months (168). It is unclear if survivors of neonatal sepsis remain at increased risk of developing subsequent infections $(31,163,169-171)$. Preterm infants may have more than one episode of sepsis ( 20\%) during their NICU admission, and it is uncertain whether having a previous episode of sepsis contributes to the overall risk compared to the major risk factor that is degree of immaturity $(31,163,169)$. Preterm neonates, remain at increased risk of infection-related admissions to hospital well into childhood (inversely related to both GA and birth weight) (172), however if neonatal sepsis contributes to infection-related hospital readmissions in childhood has not been studied. Increased risk of subsequent, more severe infections is a hallmark of SII in adults, but it is unclear if this clinical outcome is observed in neonates with sepsis.

\section{CONCLUSIONS}

Sepsis mortality in neonates may be associated with alterations in immune function that are in agreement with SII findings in adults. Whether immune cell dysfunction or impairment underpins immunosuppression in neonatal sepsis requires further investigation and stronger evidence. Large, collaborative longitudinal studies, from birth through to childhood, are essential to evaluate immune changes in neonates with sepsis, including the role for SII. Yet, first a definitive consensus on the definition of neonatal sepsis and severity needs to be established. Until then, sepsis severity could be measured by

\section{REFERENCES}

1. Fleischmann C, Scherag A, Adhikari NK, Hartog CS, Tsaganos T, Schlattmann $\mathrm{P}$, et al. Assessment of global incidence and mortality of hospital-treated sepsis. Current estimates and limitations. Am J Respir Crit Care Med. (2016) 193:259-72. doi: 10.1164/rccm.201504-0781OC

2. Singer M, Deutschman CS, Seymour CW, Shankar-Hari M, Annane D, Bauer $\mathrm{M}$, et al. The Third International Consensus Definitions for Sepsis and Septic Shock (Sepsis-3). JAMA. (2016) 315:801-10. doi: 10.1001/jama.2016.0287

3. Hotchkiss RS, Monneret G, Payen D. Sepsis-induced immunosuppression: from cellular dysfunctions to immunotherapy. Nat Rev Immunol. (2013) 13:862-74. doi: 10.1038/nri3552

4. Hotchkiss RS, Monneret G, Payen D. Immunosuppression in sepsis: a novel understanding of the disorder and a new therapeutic approach. Lancet Infect Dis. (2013) 13:260-8. doi: 10.1016/S1473-3099(13)70001-X

5. Lyle NH, Pena OM, Boyd JH, Hancock RE. Barriers to the effective treatment of sepsis: antimicrobial agents, sepsis definitions, and hostdirected therapies. Ann N Y Acad Sci. (2014) 1323:101-14. doi: 10.1111/nyas. 12444

6. Pfortmueller CA, Meisel C, Fux M, Schefold JC. Assessment of immune organ dysfunction in critical illness: utility of innate immune response markers. Intensive Care Med Exp. (2017) 5:49. doi: 10.1186/s40635-017-0163-0

7. Venet F, Monneret G. Advances in the understanding and treatment of sepsis-induced immunosuppression. Nat Rev Nephrol. (2018) 14:121-37. doi: $10.1038 /$ nrneph.2017.165 mortality. Advances in further understanding the immunological mechanisms behind immunosuppression may lead to effective targeted treatment therapies for reversing or modulating SII and improve outcomes. Immunosuppression reversal with PD1/PD-L1 antibody blockade is currently being trialled in adult cancer patients who share similar immune defects as those with SII (151). Furthermore, pentoxifylline, an immune modulatory drug, is currently being trialled to improve long-term outcomes, primarily neurodevelopment impairment, associated with neonatal sepsis (ANZCTR ACTRN12616000405415). Assessing the impact of such interventions on SII-associated markers may provide a mechanistic insight into the success or failure of these interventions in preventing short and long-term negative sepsis outcomes.

\section{AUTHOR CONTRIBUTIONS}

$\mathrm{JH}$ conceived and wrote the first and subsequent drafts. TS and AC conceived and revised the manuscript.

\section{ACKNOWLEDGMENTS}

TS is supported by a Raine Medical Research Foundation Clinician Research Fellowship.

\section{SUPPLEMENTARY MATERIAL}

The Supplementary Material for this article can be found online at: https://www.frontiersin.org/articles/10.3389/fped. 2018.00357/full\#supplementary-material

8. Arens C, Bajwa SA, Koch C, Siegler BH, Schneck E, Hecker A, et al. Sepsisinduced long-term immune paralysis-results of a descriptive, explorative study. Crit Care (2016) 20:93. doi: 10.1186/s13054-016-1233-5

9. Pena OM, Hancock DG, Lyle NH, Linder A, Russell JA, Xia J, et al. An endotoxin tolerance signature predicts sepsis and organ dysfunction at initial clinical presentation. EBioMedicine (2014) 1:64-71. doi: 10.1016/j.ebiom.2014.10.003

10. Cavaillon JM, Adib-Conquy M. Bench-to-bedside review: endotoxin tolerance as a model of leukocyte reprogramming in sepsis. Crit Care (2006) 10:233. doi: 10.1186/cc5055

11. Dobrovolskaia MA, Medvedev AE, Thomas KE, Cuesta N, Toshchakov $\mathrm{V}$, Ren $\mathrm{T}$, et al. Induction of in vitro reprogramming by Toll-like receptor (TLR)2 and TLR4 agonists in murine macrophages: effects of TLR "homotolerance" versus "heterotolerance" on NF-kappa B signaling pathway components. J Immunol. (2003) 170:508-19. doi: 10.4049/jimmunol.170.1.508

12. Levy O. Innate immunity of the newborn: basic mechanisms and clinical correlates. Nat Rev Immunol. (2007) 7:379-90. doi: 10.1038/nri2075

13. Basha S, Surendran N, Pichichero M. Immune responses in neonates. Expert Rev Clin Immunol. (2014) 10:1171-84. doi: 10.1586/1744666X.2014.9 42288

14. MacGillivray DM, Kollmann TR. The role of environmental factors in modulating immune responses in early life. Front Immunol. (2014) 5:434. doi: 10.3389/fimmu.2014.00434

15. Shah BA, Padbury JF. Neonatal sepsis: an old problem with new insights. Virulence (2014) 5:170-8. doi: 10.4161/viru.26906 
16. van Well GTJ, Daalderop LA, Wolfs T, Kramer BW. Human perinatal immunity in physiological conditions and during infection. Mol Cell Pediatr. (2017) 4:4. doi: 10.1186/s40348-017-0070-1

17. Melville JM, Moss TJ. The immune consequences of preterm birth. Front Neurosci. (2013) 7:79. doi: 10.3389/fnins.2013. 00079

18. Sharma AA, Jen R, Butler A, Lavoie PM. The developing human preterm neonatal immune system: a case for more research in this area. Clin Immunol. (2012) 145:61-8. doi: 10.1016/j.clim.2012.08.006

19. Raymond SL, Rincon JC, Wynn JL, Moldawer LL, Larson SD. Impact of early-life exposures to infections, antibiotics, and vaccines on perinatal and long-term health and disease. Front Immunol. (2017) 8:729. doi: 10.3389/fimmu.2017.00729

20. Liu L, Oza S, Hogan D, Perin J, Rudan I, Lawn JE, et al. Global, regional, and national causes of child mortality in 2000-13, with projections to inform post-2015 priorities: an updated systematic analysis. Lancet (2015) 385:430-40. doi: 10.1016/S0140-6736(14)6 1698-6

21. Greenberg RG, Kandefer S, Do BT, Smith PB, Stoll BJ, Bell EF, et al. Late-onset sepsis in extremely premature infants: 2000-2011. Pediatr Infect Dis J. (2017) 36:774-9. doi: 10.1097/INF.00000000000 01570

22. Barton L, Hodgman JE, Pavlova Z. Causes of death in the extremely low birth weight infant. Pediatrics (1999) 103:446-51. doi: 10.1542/peds.103.2.446

23. Strunk T, Inder T, Wang X, Burgner D, Mallard C, Levy O. Infectioninduced inflammation and cerebral injury in preterm infants. Lancet Infect Dis. (2014) 14:751-62. doi: 10.1016/S1473-3099(14)7 0710-8

24. Stoll BJ, Hansen NI, Adams-Chapman I, Fanaroff AA, Hintz SR, Vohr B, et al. Neurodevelopmental and growth impairment among extremely lowbirth-weight infants with neonatal infection. JAMA (2004) 292:2357-65. doi: 10.1001/jama.292.19.2357

25. Sonneville R, Verdonk F, Rauturier C, Klein IF, Wolff M, Annane D, et al. Understanding brain dysfunction in sepsis. Ann Intensive Care (2013) 3:15. doi: 10.1186/2110-5820-3-15

26. Strunk T, Currie A, Richmond P, Simmer K, Burgner D. Innate immunity in human newborn infants: prematurity means more than immaturity. J Matern Fetal Neonatal Med. (2011) 24:25-31. doi: 10.3109/14767058.2010.482605

27. Wynn JL, Wong HR. Pathophysiology and treatment of septic shock in neonates. Clin Perinatol. (2010) 37:439-79. doi: 10.1016/j.clp.2010.04.002

28. Khaertynov KS, Boichuk SV, Khaiboullina SF, Anokhin VA, Andreeva AA, Lombardi VC, et al. Comparative assessment of cytokine pattern in early and late onset of neonatal sepsis. J Immunol Res. (2017) 2017:8601063. doi: $10.1155 / 2017 / 8601063$

29. Schultz C, Rott C, Temming P, Schlenke P, Moller JC, Bucsky P. Enhanced interleukin-6 and interleukin-8 synthesis in term and preterm infants. Pediatr Res. (2002) 51:317-22. doi: 10.1203/00006450-200203000-00009

30. Rittirsch D, Flierl MA, Ward PA. Harmful molecular mechanisms in sepsis. Nat Rev Immunol. (2008) 8:776-87. doi: 10.1038/nri2402

31. Stoll BJ, Hansen N, Fanaroff AA, Wright LL, Carlo WA, Ehrenkranz RA, et al. Late-onset sepsis in very low birth weight neonates: the experience of the NICHD Neonatal Research Network. Pediatrics (2002) 110(2 Pt 1):285-91. doi: $10.1542 /$ peds.110.2.285

32. Stoll BJ, Hansen NI, Sanchez PJ, Faix RG, Poindexter BB, Van Meurs KP, et al. Early onset neonatal sepsis: the burden of group B Streptococcal and E. coli disease continues. Pediatrics (2011) 127:817-26. doi: $10.1542 /$ peds.2010-2217

33. Wynn JL, Wilson CS, Hawiger J, Scumpia PO, Marshall AF, Liu JH, et al. Targeting IL-17A attenuates neonatal sepsis mortality induced by IL-18. Proc Natl Acad Sci USA. (2016) 113:E2627-35. doi: 10.1073/pnas.1515793113

34. Roger T, Schneider A, Weier M, Sweep FC, Le Roy D, Bernhagen J, et al. High expression levels of macrophage migration inhibitory factor sustain the innate immune responses of neonates. Proc Natl Acad Sci USA. (2016) 113:E997-1005. doi: 10.1073/pnas.1514018113

35. Maddux AB, Douglas IS. Is the developmentally immature immune response in paediatric sepsis a recapitulation of immune tolerance? Immunology (2015) 145:1-10. doi: 10.1111/imm.12454
36. Cortese F, Scicchitano P, Gesualdo M, Filaninno A, De Giorgi E, Schettini F, et al. Early and late infections in newborns: where do we stand? A review. Pediatr Neonatol. (2016) 57:265-73. doi: 10.1016/j.pedneo.2015.09.007

37. Vincent JL, Rello J, Marshall J, Silva E, Anzueto A, Martin CD, et al. International study of the prevalence and outcomes of infection in intensive care units. JAMA. (2009) 302:2323-9. doi: 10.1001/jama.2009.1754

38. Gervassi AL, Horton H. Is infant immunity actively suppressed or immature? Virology (2014) 2014:1-9. doi: 10.4137/VRT.S12248

39. Goldstein B, Giroir B, Randolph A. International pediatric sepsis consensus conference: definitions for sepsis and organ dysfunction in pediatrics. Pediatr Crit Care Med. (2005) 6:2-8. doi: 10.1097/00130478-200501000-00049

40. Hofer N, Zacharias E, Muller W, Resch B. Performance of the definitions of the systemic inflammatory response syndrome and sepsis in neonates. $J$ Perinat Med. (2012) 40:587-90. doi: 10.1515/jpm-2011-0308

41. Wynn JL. Defining neonatal sepsis. Curr Opin Pediatr. (2016) 28:135-40. doi: 10.1097/MOP.0000000000000315

42. Wynn JL, Wong HR, Shanley TP, Bizzarro MJ, Saiman L, Polin RA. Time for a neonatal-specific consensus definition for sepsis. Pediatr Crit Care Med. (2014) 15:523-8. doi: 10.1097/PCC.0000000000000157

43. Ng S, Strunk T, Jiang P, Muk T, Sangild PT, Currie A. Precision medicine for neonatal sepsis. Front Mol Biosci. (2018) 5:70. doi: 10.3389/fmolb.2018.00070

44. Meem M, Modak JK, Mortuza R, Morshed M, Islam MS, Saha SK. Biomarkers for diagnosis of neonatal infections: a systematic analysis of their potential as a point-of-care diagnostics. J Glob Health (2011) 1:201-9.

45. Dong Y, Speer CP. Late-onset neonatal sepsis: recent developments. Arch Dis Child Fetal Neonatal Ed. (2015) 100:F257-63. doi: 10.1136/archdischild-2014-306213

46. Melvan JN, Bagby GJ, Welsh DA, Nelson S, Zhang P. Neonatal sepsis and neutrophil insufficiencies. Int Rev Immunol. (2010) 29:315-48. doi: 10.3109/08830181003792803

47. Guilmot A, Hermann E, Braud VM, Carlier Y, Truyens C. Natural killer cell responses to infections in early life. J Innate Immun. (2011) 3:280-8. doi: 10.1159/000323934

48. Lawrence SM, Eckert J, Makoni M, Pereira HA. Is the use of complete blood counts with manual differentials an antiquated method of determining neutrophil composition in newborns? Ann Clin Lab Sci. (2015) 45:403-13.

49. Simon AK, Hollander GA, McMichael A. Evolution of the immune system in humans from infancy to old age. Proc Biol Sci. (2015) 282:20143085. doi: $10.1098 / \mathrm{rspb} .2014 .3085$

50. Camacho-Gonzalez A, Spearman PW, Stoll BJ. Neonatal infectious diseases: evaluation of neonatal sepsis. Pediatr Clin North Am. (2013) 60:367-89. doi: 10.1016/j.pcl.2012.12.003

51. Maddux AB, Hiller TD, Overdier KH, Pyle LL, Douglas IS. Innate immune function and organ failure recovery in adults with sepsis. I Intensive Care Med. 2017:885066617701903. doi: 10.1177/0885066617701903

52. Monneret G, Finck ME, Venet F, Debard AL, Bohe J, Bienvenu J, et al. The anti-inflammatory response dominates after septic shock: association of low monocyte HLA-DR expression and high interleukin-10 concentration. Immunol Lett. (2004) 95:193-8. doi: 10.1016/j.imlet.2004.07.009

53. Silveira-Lessa AL, Quinello C, Lima L, Redondo ACC, Ceccon M, Carneiro-Sampaio M, et al. TLR expression, phagocytosis and oxidative burst in healthy and septic newborns in response to Gramnegative and Gram-positive rods. Hum. Immunol. (2016) 77:972-80. doi: 10.1016/j.humimm.2016.07.230

54. Prashant A, Vishwanath P, Kulkarni P, Sathya Narayana P, Gowdara V, Nataraj SM, et al. Comparative assessment of cytokines and other inflammatory markers for the early diagnosis of neonatal sepsis-a case control study. PLoS ONE (2013) 8:e68426. doi: 10.1371/journal.pone.0068426

55. Kocabas E, Sarikcioglu A, Aksaray N, Seydaoglu G, Seyhun Y, Yaman A. Role of procalcitonin, C-reactive protein, interleukin-6, interleukin-8 and tumor necrosis factor-alpha in the diagnosis of neonatal sepsis. Turk J Pediatr. (2007) 49:7-20.

56. Tamayo E, Fernandez A, Almansa R, Carrasco E, Heredia M, Lajo C, et al. Pro- and anti-inflammatory responses are regulated simultaneously from the first moments of septic shock. Eur Cytokine Netw. (2011) 22:82-7. doi: 10.1684/ecn.2011.0281 
57. Ono S, Kimura A, Hiraki S, Takahata R, Tsujimoto H, Kinoshita $\mathrm{M}$, et al. Removal of increased circulating CD4+CD25+Foxp3+ regulatory $\mathrm{T}$ cells in patients with septic shock using hemoperfusion with polymyxin B-immobilized fibers. Surgery (2013) 153:262-71. doi: 10.1016/j.surg.2012.06.023

58. van Dissel JT, van Langevelde P, Westendorp RG, Kwappenberg K, Frolich M. Anti-inflammatory cytokine profile and mortality in febrile patients. Lancet (1998) 351:950-3. doi: 10.1016/S0140-6736(05)6 0606-X

59. Hynninen M, Pettila V, Takkunen O, Orko R, Jansson SE, Kuusela P, et al. Predictive value of monocyte histocompatibility leukocyte antigenDR expression and plasma interleukin-4 and-10 levels in critically ill patients with sepsis. Shock (2003) 20:1-4. doi: 10.1097/01.shk.0000068322.08 268.b4

60. Weighardt $H$, Heidecke CD, Emmanuilidis K, Maier S, Bartels H, Siewert JR, et al. Sepsis after major visceral surgery is associated with sustained and interferon-gamma-resistant defects of monocyte cytokine production. Surgery (2000) 127:309-15. doi: 10.1067/msy.2000.1 04118

61. Papadopoulos P, Pistiki A, Theodorakopoulou M, Christodoulopoulou T, Damoraki G, Goukos D, et al. Immunoparalysis: clinical and immunological associations in SIRS and severe sepsis patients. Cytokine (2017) 92:83-92. doi: 10.1016/j.cyto.2017.01.012

62. Winkler MS, Rissiek A, Priefler M, Schwedhelm E, Robbe L, Bauer A, et al. Human leucocyte antigen (HLA-DR) gene expression is reduced in sepsis and correlates with impaired TNF $\alpha$ response: a diagnostic tool for immunosuppression? PLoS ONE (2017) 12:e0182427. doi: 10.1371/journal.pone.0182427

63. Manjuck J, Saha DC, Astiz M, Eales LJ, Rackow EC. Decreased response to recall antigens is associated with depressed costimulatory receptor expression in septic critically ill patients. J Lab Clin Med. (2000) 135:153-60. doi: $10.1067 / \mathrm{mlc} .2000 .104306$

64. Genel F, Atlihan F, Ozsu E, Ozbek E. Monocyte HLA-DR expression as predictor of poor outcome in neonates with late onset neonatal sepsis. $J$ Infect. (2010) 60:224-8. doi: 10.1016/j.jinf.2009.12.004

65. Pradhan R, Jain P, Paria A, Saha A, Sahoo J, Sen A, et al. Ratio of neutrophilic CD64 and monocytic HLA-DR: a novel parameter in diagnosis and prognostication of neonatal sepsis. Cytometry B Clin Cytom. (2016) 90:295-302. doi: 10.1002/cyto.b.21244

66. Fotopoulos S, Debonera F, Stamokosta E, Paterakis G, Stavropoulos-Giokas C, Germenis A, et al., (eds.). The monocyte HLA-DR expression of healthy and infected premature neonates. Pediatr Res (1999) 45:763.

67. Guerin E, Orabona M, Raquil MA, Giraudeau B, Bellier $\mathrm{R}$, Gibot $\mathrm{S}$, et al. Circulating immature granulocytes with T-cell killing functions predict sepsis deterioration*. Crit Care Med. (2014) 42:2007-18. doi: 10.1097/CCM.0000000000 000344

68. Mare TA, Treacher DF, Shankar-Hari M, Beale R, Lewis SM, Chambers DJ, et al. The diagnostic and prognostic significance of monitoring blood levels of immature neutrophils in patients with systemic inflammation. Crit Care (2015) 19:57. doi: 10.1186/s13054-015-0778-z

69. Demaret J, Venet F, Friggeri A, Cazalis MA, Plassais J, Jallades L, et al. Marked alterations of neutrophil functions during sepsisinduced immunosuppression. J Leukoc Biol. (2015) 98:1081-90. doi: 10.1189/jlb.4A0415-168RR

70. Saied DA. Can we rely on the neutrophil left shift for the diagnosis of neonatal sepsis? Need for re-evaluation. Egypt Pediatr Assoc Gaz. (2018) 66. doi: 10.1016/j.epag.2017.12.002

71. Itoh $\mathrm{K}$, Aihara $\mathrm{H}$, Takada S, Nishino $\mathrm{M}$, Lee $\mathrm{Y}$, Negishi $\mathrm{H}$, et al. Clinicopathological differences between early-onset and late-onset sepsis and pneumonia in very low birth weight infants. Pediatr Pathol. (1990) 10:757-68. doi: 10.3109/15513819009064710

72. Li J, Li M, Su L, Wang H, Xiao K, Deng J, et al. Alterations of $\mathrm{T}$ helper lymphocyte subpopulations in sepsis, severe sepsis, and septic shock: a prospective observational study. Inflammation (2015) 38:995-1002. doi: 10.1007/s10753-014-0063-3

73. Monneret G, Debard AL, Venet F, Bohe J, Hequet O, Bienvenu J, et al. Marked elevation of human circulating CD $4+\mathrm{CD} 25+$ regulatory $\mathrm{T}$ cells in sepsis-induced immunoparalysis. Crit Care Med. (2003) 31:2068-71. doi: 10.1097/01.CCM.0000069345.78884.0F

74. Liu Q, An L, Qi Z, Zhao Y, Li C. Increased expression of programmed cell death-1 in regulatory $\mathrm{T}$ cells of patients with severe sepsis and septic shock: an observational clinical study. Scand J Immunol. (2017) 86:408-17. doi: $10.1111 /$ sji.12612

75. Huang H, Xu R, Lin F, Bao C, Wang S, Ji C, et al. High circulating CD39(+) regulatory $\mathrm{T}$ cells predict poor survival for sepsis patients. Int J Infect Dis. (2015) 30:57-63. doi: 10.1016/j.ijid.2014.11.006

76. Uhel F, Azzaoui I, Gregoire M, Pangault C, Dulong J, Tadie JM, et al. Early expansion of circulating granulocytic myeloid-derived suppressor cells predicts development of nosocomial infections in patients with sepsis. Am J Respir Crit Care Med. (2017) 196:315-27. doi: 10.1164/rccm.201606-1143OC

77. Mathias B, Delmas AL, Ozrazgat-Baslanti T, Vanzant EL, Szpila BE, Mohr $\mathrm{AM}$, et al. Human myeloid-derived suppressor cells are associated with chronic immune suppression after severe sepsis/septic shock. Ann Surg. (2017) 265:827-34. doi: 10.1097/SLA.0000000000001783

78. Darcy CJ, Minigo G, Piera KA, Davis JS, McNeil YR, Chen Y, et al. Neutrophils with myeloid derived suppressor function deplete arginine and constrain T cell function in septic shock patients. Crit. Care (2014) 18:R163. doi: 10.1186/cc14003

79. Guignant C, Lepape A, Huang X, Kherouf H, Denis L, Poitevin F, et al. Programmed death-1 levels correlate with increased mortality, nosocomial infection and immune dysfunctions in septic shock patients. Crit Care (2011) 15:R99. doi: 10.1186/cc10112

80. Shao R, Fang Y, Yu H, Zhao L, Jiang Z, Li CS. Monocyte programmed death ligand-1 expression after 3-4 days of sepsis is associated with risk stratification and mortality in septic patients: a prospective cohort study. Crit Care (2016) 20:124. doi: 10.1186/s13054-016-1301-x

81. Zasada M, Lenart M, Rutkowska-Zapala M, Stec M, Durlak W, Grudzien A, et al. Analysis of PD-1 expression in the monocyte subsets from non-septic and septic preterm neonates. PLoS ONE. (2017) 12:e0186819. doi: 10.1371/journal.pone.0186819

82. Hotchkiss RS, Osmon SB, Chang KC, Wagner TH, Coopersmith CM, Karl IE. Accelerated lymphocyte death in sepsis occurs by both the death receptor and mitochondrial pathways. J Immunol. (2005) 174:5110-8. doi: 10.4049/jimmunol.174.8.5110

83. Zhang Y, Li J, Lou J, Zhou Y, Bo L, Zhu J, et al. Upregulation of programmed death-1 on $\mathrm{T}$ cells and programmed death ligand-1 on monocytes in septic shock patients. Crit Care (2011) 15:R70. doi: 10.1186/cc 10059

84. Boomer JS, To K, Chang KC, Takasu O, Osborne DF, Walton AH, et al. Immunosuppression in patients who die of sepsis and multiple organ failure. JAMA. (2011) 306:2594-605. doi: 10.1001/jama.2011.1829

85. Hotchkiss RS, Tinsley KW, Swanson PE, Schmieg RE, Jr., Hui JJ, Chang $\mathrm{KC}$, et al. Sepsis-induced apoptosis causes progressive profound depletion of B and CD4+ T lymphocytes in humans. J Immunol. (2001) 166:6952-63. doi: 10.4049/jimmunol.166.11.6952

86. Christensen RD, Rothstein G. Exhaustion of mature marrow neutrophils in neonates with sepsis. J Pediatr. (1980) 96:316-8. doi: 10.1016/S0022-3476(80)80837-7

87. Toti P, De Felice C, Occhini R, Schuerfeld K, Stumpo M, Epistolato MC, et al. Spleen depletion in neonatal sepsis and chorioamnionitis. Am J Clin Pathol. (2004) 122:765-71. doi: 10.1309/RV6E9BMC9954A2WU

88. Striz I, Brabcova E, Kolesar L, Sekerkova A. Cytokine networking of innate immunity cells: a potential target of therapy. Clin Sci. (2014) 126:593-612. doi: 10.1042/CS20130497

89. Munoz C, Carlet J, Fitting C, Misset B, Bleriot JP, Cavaillon JM. Dysregulation of in vitro cytokine production by monocytes during sepsis. J Clin Invest. (1991) 88:1747-54. doi: 10.1172/JCI115493

90. Rigato O, Salomao R. Impaired production of interferon-gamma and tumor necrosis factor-alpha but not of interleukin 10 in whole blood of patients with sepsis. Shock (2003) 19:113-6. doi: 10.1097/00024382-200302000-00004

91. Sinistro A, Almerighi C, Ciaprini C, Natoli S, Sussarello E, Di Fino S, et al. Downregulation of CD40 ligand response in monocytes from sepsis patients. Clin Vacc Immunol. (2008) 15:1851-8. doi: 10.1128/CVI.00184-08

92. Segura-Cervantes E, Mancilla-Ramirez J, Gonzalez-Canudas J, Alba E, Santillan-Ballesteros R, Morales-Barquet D, et al. Inflammatory response in 
preterm and very preterm newborns with sepsis. Mediat inflamm. (2016) 2016:6740827. doi: 10.1155/2016/6740827

93. Drossou-Agakidou V, Kanakoudi-Tsakalidou F, Sarafidis K, Tzimouli V, Taparkou A, Kremenopoulos G, et al. In vivo effect of rhGM-CSF And rhGCSF on monocyte HLA-DR expression of septic neonates. Cytokine (2002) 18:260-5. doi: 10.1006/cyto.2002.1037

94. Ng PC, Li K, Wong RP, Chui K, Wong E, Li G, et al. Proinflammatory and anti-inflammatory cytokine responses in preterm infants with systemic infections. Arch Dis Child Fetal Neonatal Ed. (2003) 88:F209-13. doi: 10.1136/fn.88.3.F209

95. Ye Q, Du LZ, Shao WX, Shang SQ. Utility of cytokines to predict neonatal sepsis. Pediatr Res. (2017) 81:616-21. doi: 10.1038/pr.2016.267

96. Silveira RC, Procianoy RS. Evaluation of interleukin-6, tumour necrosis factor-alpha and interleukin-1beta for early diagnosis of neonatal sepsis. Acta Paediatr. (1999) 88:647-50. doi: 10.1080/080352599501 69314

97. Engelberts I, Stephens S, Francot GJ, van der Linden CJ, Buurman WA. Evidence for different effects of soluble TNF-receptors on various TNF measurements in human biological fluids. Lancet (1991) 338:515-6. doi: 10.1016/0140-6736(91)90591-C

98. van Deuren M. Kinetics of tumour necrosis factor-alpha, soluble tumour necrosis factor receptors, interleukin 1-beta and its receptor antagonist during serious infections. Eur J Clin Microbiol Infect Dis. (1994) 13(Suppl. 1):S12-6. doi: 10.1007/BF02390680

99. Afonso EDP, Blot S. Effect of gestational age on the epidemiology of lateonset sepsis in neonatal intensive care units - a review. Expert Rev Anti Infect. Ther. (2017) 15:917-24. doi: 10.1080/14787210.2017.1379394

100. Jekarl DW, Lee SY, Lee J, Park YJ, Kim Y, Park JH, et al. Procalcitonin as a diagnostic marker and IL-6 as a prognostic marker for sepsis. Diagn Microbiol Infect Dis. (2013) 75:342-7. doi: 10.1016/j.diagmicrobio.2012.12.011

101. Barre M, Behnes M, Hamed S, Pauly D, Lepiorz D, Lang S, et al. Revisiting the prognostic value of monocyte chemotactic protein 1 and interleukin6 in the sepsis-3 era. J Crit Care (2018) 43:21-8. doi: 10.1016/j.jcrc.2017. 08.024

102. Zasada M, Lenart M, Rutkowska-Zapala M, Stec M, Mol N, Czyz $\mathrm{O}$, et al. Analysis of selected aspects of inflammasome function in the monocytes from neonates born extremely and very prematurely. Immunobiology (2018) 223:18-24. doi: 10.1016/j.imbio.2017. 10.019

103. Parihar A, Eubank TD, Doseff AI. Monocytes and macrophages regulate immunity through dynamic networks of survival and cell death. J Innate Immun. (2010) 2:204-15. doi: 10.1159/0002 96507

104. Hamers L, Kox M, Pickkers P. Sepsis-induced immunoparalysis: mechanisms, markers, and treatment options. Mine Anestesiol. (2015) 814)426-39.

105. Sundar KM, Sires M. Sepsis induced immunosuppression: implications for secondary infections and complications. Ind J Crit Care Med. (2013) 17:162-9. doi: 10.4103/0972-5229.117054

106. Docke WD, Hoflich C, Davis KA, Rottgers K, Meisel C, Kiefer P, et al. Monitoring temporary immunodepression by flow cytometric measurement of monocytic HLA-DR expression: a multicenter standardized study. Clin Chem. (2005) 51:2341-7. doi: 10.1373/clinchem.2005.052639

107. Frazier WJ, Hall MW. Immunoparalysis and adverse outcomes from critical illness. Pediatr Clin North Am. (2008) 55:647-68, xi. doi: 10.1016/j.pcl.2008.02.009

108. Lukaszewicz AC, Grienay M, Resche-Rigon M, Pirracchio R, Faivre V, Boval $\mathrm{B}$, et al. Monocytic HLA-DR expression in intensive care patients: interest for prognosis and secondary infection prediction. Crit Care Med. (2009) 37:2746-52. doi: 10.1097/CCM.0b013e3181ab858a

109. de Waal Malefyt R, Haanen J, Spits H, Roncarolo MG, te Velde A, Figdor C, et al. Interleukin 10 (IL-10) and viral IL-10 strongly reduce antigen-specific human $\mathrm{T}$ cell proliferation by diminishing the antigen-presenting capacity of monocytes via downregulation of class II major histocompatibility complex expression. J Exp Med. (1991) 174:915-24. doi: 10.1084/jem.174.4.915

110. Koppelman B, Neefjes JJ, de Vries JE, de Waal Malefyt R. Interleukin-10 down-regulates MHC class II alphabeta peptide complexes at the plasma membrane of monocytes by affecting arrival and recycling. Immunity (1997) 7:861-71. doi: 10.1016/S1074-7613(00)80404-5

111. Fumeaux T, Pugin J. Role of interleukin-10 in the intracellular sequestration of human leukocyte antigen-DR in monocytes during septic shock. Am J Respir Crit Care Med. (2002) 166:1475-82. doi: 10.1164/rccm.200203217OC

112. Kanakoudi-Tsakalidou F, Debonera F, Drossou-Agakidou V, Sarafidis K, Tzimouli V, Taparkou A, et al. Flow cytometric measurement of HLADR expression on circulating monocytes in healthy and sick neonates using monocyte negative selection. Clin Exp Immunol. (2001) 123:402-7. doi: 10.1046/j.1365-2249.2001.01471.x

113. Palojarvi A, Petaja J, Siitonen S, Janer C, Andersson S. Low monocyte HLADR expression as an indicator of immunodepression in very low birth weight infants. Pediatr Res. (2013) 73(4 Pt 1):469-75. doi: 10.1038/pr.2012.199

114. Juskewitch JE, Abraham RS, League SC, Jenkins SM, Smith CY, Enders FT, et al. Monocyte HLA-DR expression and neutrophil CD64 expression as biomarkers of infection in critically ill neonates and infants. Pediatr Res. (2015) 78:683-90. doi: 10.1038/pr.2015.164

115. Skrzeczynska J, Kobylarz K, Hartwich Z, Zembala M, Pryjma J. CD14+CD16+ monocytes in the course of sepsis in neonates and small children: monitoring and functional studies. Scand J Immunol. (2002) 556):629-38. doi: 10.1046/j.1365-3083.2002.01092.x

116. Pillay J, Kamp VM, van Hoffen E, Visser T, Tak T, Lammers JW, et al. A subset of neutrophils in human systemic inflammation inhibits $\mathrm{T}$ cell responses through Mac-1. J Clin Invest. (2012) 122:327-36. doi: 10.1172/JCI57990

117. Newman TB, Draper D, Puopolo KM, Wi S, Escobar GJ. Combining immature and total neutrophil counts to predict early onset sepsis in term and late preterm newborns: use of the I/T2. Pediatr Infect Dis J. (2014) 33:798-802. doi: 10.1097/INF.0000000000000297

118. Mikhael M, Brown LS, Rosenfeld CR. Serial neutrophil values facilitate predicting the absence of neonatal early-onset sepsis. J Pediatr. (2014) 164:522 -528.e3. doi: 10.1016/j.jpeds.2013.10.080

119. Murphy K, Weiner J. Use of leukocyte counts in evaluation of early-onset neonatal sepsis. Pediatr Infect Dis J. (2012) 31:16-9. doi: 10.1097/INF.0b013e31822ffc17

120. Cimenti C, Erwa W, Herkner KR, Kasper DC, Muller W, Resch B. The predictive value of immature granulocyte count and immature myeloid information in the diagnosis of neonatal sepsis. Clin Chem Lab Med. (2012) 50:1429-32. doi: 10.1515/cclm-2011-0656

121. Hornik CP, Benjamin DK, Becker KC, Benjamin DK, Jr., Li J, Clark RH, et al. Use of the complete blood cell count in late-onset neonatal sepsis. Pediatr Infect Dis J. (2012) 31:803-7. doi: 10.1097/INF.0b013e31825691e4

122. Cao C, Ma T, Chai YF, Shou ST. The role of regulatory $\mathrm{T}$ cells in immune dysfunction during sepsis. World J Emerg Med. (2015) 6:5-9. doi: 10.5847/wjem.j.1920-8642.2015.01.001

123. Wan YY. Regulatory T cells: immune suppression and beyond. Cell Mol Immunol. (2010) 7:204-10. doi: 10.1038/cmi.2010.20

124. Dieckmann D, Plottner H, Berchtold S, Berger T, Schuler G. Ex vivo isolation and characterization of $\mathrm{CD} 4(+) \mathrm{CD} 25(+) \mathrm{T}$ cells with regulatory properties from human blood. J Exp Med. (2001) 193:1303-10. doi: 10.1084/jem.193.11.1303

125. Schmidt A, Oberle N, Krammer PH. Molecular mechanisms of treg-mediated $\mathrm{T}$ cell suppression. Front Immunol. (2012) 3:51. doi: 10.3389/fimmu.2012.00051

126. Sakaguchi S, Wing K, Onishi Y, Prieto-Martin P, Yamaguchi T. Regulatory T cells: how do they suppress immune responses? Int Immunol. (2009) 21:1105-11. doi: 10.1093/intimm/dxp095

127. Levings MK, Sangregorio R, Roncarolo MG. Human cd25(+)cd4(+) t regulatory cells suppress naive and memory $\mathrm{T}$ cell proliferation and can be expanded in vitro without loss of function. J Exp Med. (2001) 193:1295-302. doi: 10.1084/jem.193.11.1295

128. Parry RV, Chemnitz JM, Frauwirth KA, Lanfranco AR, Braunstein I, Kobayashi SV, et al. CTLA-4 and PD-1 receptors inhibit T-cell activation by distinct mechanisms. Mol Cell Biol. (2005) 25:9543-53. doi: 10.1128/MCB.25.21.9543-9553.2005

129. Ghiringhelli F, Menard C, Terme M, Flament C, Taieb J, Chaput $\mathrm{N}$, et al. CD4+CD25+ regulatory $\mathrm{T}$ cells inhibit natural killer cell functions in a transforming growth factor-beta-dependent 
manner. J Exp Med. (2005) 202:1075-85. doi: 10.1084/jem.200 51511

130. Taams LS, van Amelsfort JM, Tiemessen MM, Jacobs KM, de Jong EC, Akbar AN, et al. Modulation of monocyte/macrophage function by human CD4+CD25+ regulatory T cells. Hum Immunol. (2005) 66:222-30. doi: 10.1016/j.humimm.2004.12.006

131. Lim HW, Hillsamer P, Banham AH, Kim CH. Cutting edge: direct suppression of B cells by CD4+ CD25+ regulatory T cells. J Immunol. (2005) 175:4180-3. doi: 10.4049/jimmunol.175.7.4180

132. Behrendt J, Karpe J, Mazur B, Stojewska M, Sadownik B, Wasek A, et al. [Quantitative changes of CD3+, CD4+, CD8+, HLA-DR+, T lymphocytes and CD25+ cells in eutrophic full-term neonates with staphylococcal septicemia]. Przegl lek. (2002) 59(Suppl. 1):50-3.

133. Pagel J, Hartz A, Figge J, Gille C, Eschweiler S, Petersen K, et al. Regulatory $\mathrm{T}$ cell frequencies are increased in preterm infants with clinical earlyonset sepsis. Clin Exp Immunol. (2016) 185:219-27. doi: 10.1111/cei. 12810

134. Takahata Y, Nomura A, Takada H, Ohga S, Furuno K, Hikino S, et al. CD25+CD4+ T cells in human cord blood: an immunoregulatory subset with naive phenotype and specific expression of forkhead box $\mathrm{p} 3$ (Foxp3) gene. Exp Hematol. (2004) 32:622-9. doi: 10.1016/j.exphem.2004. 03.012

135. Dirix V, Vermeulen F, Mascart F. Maturation of CD4+ regulatory $\mathrm{T}$ lymphocytes and of cytokine secretions in infants born prematurely. J Clin. Immunol. (2013) 33:1126-33. doi: 10.1007/s10875-0139911-4

136. Luciano AA, Arbona-Ramirez IM, Ruiz R, Llorens-Bonilla BJ, Martinez-Lopez DG, Funderburg $\mathrm{N}$, et al. Alterations in regulatory $\mathrm{T}$ cell subpopulations seen in preterm infants. PLoS ONE (2014) 9:e95867. doi: 10.1371/journal.pone.00 95867

137. Rueda CM, Moreno-Fernandez ME, Jackson CM, Kallapur SG, Jobe $\mathrm{AH}$, Chougnet CA. Neonatal regulatory $\mathrm{T}$ cells have reduced capacity to suppress dendritic cell function. Eur J Immunol. (2015) 45:2582-92. doi: 10.1002/eji.201445371

138. Janols H, Bergenfelz C, Allaoui R, Larsson AM, Ryden L, Bjornsson S, et al. A high frequency of MDSCs in sepsis patients, with the granulocytic subtype dominating in gram-positive cases. J Leukoc Biol. (2014) 96:685-93. doi: 10.1189/jlb.5HI0214-074R

139. Schwarz J, Scheckenbach V, Kugel H, Spring B, Pagel J, Hartel C, et al. Granulocytic myeloid-derived suppressor cells (GR-MDSC) accumulate in cord blood of preterm infants and remain elevated during the neonatal period. Clin Exp Immunol. (2018) 191:328-37. doi: 10.1111/cei.13059

140. El Gazzar M. Immunobiology of S100A8 and S100A9 proteins and their role in acute inflammation and sepsis. Int J Immunol Immunother. (2015) 2:2. doi: 10.23937/2378-3672/1410013

141. Cheng P, Corzo CA, Luetteke N, Yu B, Nagaraj S, Bui MM, et al. Inhibition of dendritic cell differentiation and accumulation of myeloid-derived suppressor cells in cancer is regulated by S100A9 protein. J Exp Med. (2008) 205:2235-49. doi: 10.1084/jem.200 80132

142. Sinha P, Okoro C, Foell D, Freeze HH, Ostrand-Rosenberg S, Srikrishna G. Proinflammatory S100 proteins regulate the accumulation of myeloid-derived suppressor cells. J Immunol. (2008) 181:4666-75. doi: 10.4049/jimmunol.181.7.4666

143. Austermann J, Friesenhagen J, Fassl SK, Petersen B, Ortkras T, Burgmann J, et al. Alarmins MRP8 and MRP14 induce stress tolerance in phagocytes under sterile inflammatory conditions. Cell Rep. (2014) 9:2112-23. doi: 10.1016/j.celrep.2014.11.020

144. Decembrino L, De Amici M, Pozzi M, De Silvestri A, Stronati M. Serum calprotectin: a potential biomarker for neonatal sepsis. J Immunol Res. (2015) 2015:147973. doi: $10.1155 / 2015 / 147973$

145. Brahmamdam P, Inoue S, Unsinger J, Chang KC, McDunn JE, Hotchkiss RS. Delayed administration of anti-PD-1 antibody reverses immune dysfunction and improves survival during sepsis. J Leukoc Biol. (2010) 88:233-40. doi: 10.1189/jlb.0110037

146. Chang K, Svabek C, Vazquez-Guillamet C, Sato B, Rasche D, Wilson S, et al. Targeting the programmed cell death 1: programmed cell death ligand 1 pathway reverses $\mathrm{T}$ cell exhaustion in patients with sepsis. Crit Care (2014) 18:R3. doi: $10.1186 / \mathrm{cc} 13176$

147. de Kleijn S, Langereis JD, Leentjens J, Kox M, Netea MG, Koenderman $\mathrm{L}$, et al. IFN-gamma-stimulated neutrophils suppress lymphocyte proliferation through expression of PD-L1. PLoS ONE (2013) 8:e72249. doi: 10.1371/journal.pone.0072249

148. Patil NK, Guo Y, Luan L, Sherwood ER. Targeting immune cell checkpoints during sepsis. Int J Mol Sci. (2017) 18:2413. doi: 10.3390/ijms18112413

149. Zhang Y, Zhou Y, Lou J, Li J, Bo L, Zhu K, et al. PD-L1 blockade improves survival in experimental sepsis by inhibiting lymphocyte apoptosis and reversing monocyte dysfunction. Crit Care (2010) 14:R220. doi: $10.1186 /$ cc9354

150. Huang X, Venet F, Wang YL, Lepape A, Yuan Z, Chen Y, et al. PD-1 expression by macrophages plays a pathologic role in altering microbial clearance and the innate inflammatory response to sepsis. Proc Natl Acad Sci USA. (2009) 106:6303-8. doi: 10.1073/pnas.08094 22106

151. Topalian SL, Hodi FS, Brahmer JR, Gettinger SN, Smith DC, McDermott DF, et al. Safety, activity, and immune correlates of anti-PD-1 antibody in cancer. New Engl J Med. (2012) 366:2443-54. doi: 10.1056/NEJMoa1200690

152. Young WA, Fallon EA, Heffernan DS, Efron PA, Cioffi WG, Ayala A. Improved survival after induction of sepsis by cecal slurry in PD-1 knockout murine neonates. Surgery (2017) 161:1387-93. doi: 10.1016/j.surg.2016.11.008

153. Markel GS, Y.; Mandel, I.; Hakim, M.; Shaked, R.;. Inhibition of the novel immune checkpoint CEACAM1 to enhance anti-tumor immunological activity. J Clin Oncol. (2016) 34(15 Suppl.):3044. doi: 10.1200/JCO.2016.34.15_suppl.3044

154. Chen CJ, Shively JE. The cell-cell adhesion molecule carcinoembryonic antigen-related cellular adhesion molecule 1 inhibits IL-2 production and proliferation in human $\mathrm{T}$ cells by association with Src homology protein1 and down-regulates IL-2 receptor. J Immunol. (2004) 172:3544-52. doi: 10.4049/jimmunol.172.6.3544

155. van der Flier M, Sharma DB, Estevao S, Emonts M, Rook D, Hazelzet JA, et al. Increased CD4(+) T cell co-inhibitory immune receptor CEACAM1 in neonatal sepsis and soluble-CEACAM1 in meningococcal sepsis: a role in sepsis-associated immune suppression? PLoS ONE (2013) 8:e68294. doi: 10.1371/journal.pone.0068294

156. Hotchkiss RS, Swanson PE, Freeman BD, Tinsley KW, Cobb JP, Matuschak GM, et al. Apoptotic cell death in patients with sepsis, shock, and multiple organ dysfunction. Crit Care Med. (1999) 27:1230-51. doi: 10.1097/00003246-199907000-00002

157. Hotchkiss RS, Tinsley KW, Swanson PE, Grayson MH, Osborne DF, Wagner $\mathrm{TH}$, et al. Depletion of dendritic cells, but not macrophages, in patients with sepsis. J Immunol. (2002) 168:2493-500. doi: 10.4049/jimmunol.168.5.2493

158. Hotchkiss RS, Swanson PE, Knudson CM, Chang KC, Cobb JP, Osborne $\mathrm{DF}$, et al. Overexpression of Bcl-2 in transgenic mice decreases apoptosis and improves survival in sepsis. J Immunol. (1999) 162:4 148-56.

159. Hotchkiss RS, Chang KC, Grayson MH, Tinsley KW, Dunne BS, Davis CG, et al. Adoptive transfer of apoptotic splenocytes worsens survival, whereas adoptive transfer of necrotic splenocytes improves survival in sepsis. Proc Natl Acad Sci USA. (2003) 100:6724-9. doi: 10.1073/pnas.10317 88100

160. Inoue S, Unsinger J, Davis CG, Muenzer JT, Ferguson TA, Chang K, et al. IL-15 prevents apoptosis, reverses innate and adaptive immune dysfunction, and improves survival in sepsis. J Immunol. (2010) 184:1401-9. doi: 10.4049/jimmunol.0902307

161. Meng Y, Zhao Z, Zhu W, Yang T, Deng X, Bao R. CD155 blockade improves survival in experimental sepsis by reversing dendritic cell dysfunction. Biochem Biophys Res Commun. (2017) 490:283-9. doi: 10.1016/j.bbrc.2017.06.037

162. Bolognese AC, Yang WL, Hansen LW, Denning NL, Nicastro JM, Coppa GF, et al. Inhibition of necroptosis attenuates lung injury and improves survival in neonatal sepsis. Surgery. (2018) 2018:S0039-6060(18)30096-5. doi: 10.1016/j.surg.2018.02.017

163. Tsai MH, Hsu JF, Chu SM, Lien R, Huang HR, Chiang MC, et al. Incidence, clinical characteristics and risk factors for adverse outcome in 
neonates with late-onset sepsis. Pediatr Infect Dis J. (2014) 33:e7-e13. doi: 10.1097/INF.0b013e3182a72ee0

164. Qazi SA, Stoll BJ. Neonatal sepsis: a major global public health challenge. Pediatr Infect Dis J. (2009) 28(1 Suppl.):S1-2. doi: 10.1097/INF.0b013e31819587a9

165. Liu L, Johnson HL, Cousens S, Perin J, Scott S, Lawn JE, et al. Global, regional, and national causes of child mortality: an updated systematic analysis for 2010 with time trends since 2000. Lancet (2012) 379:2151-61. doi: 10.1016/S0140-6736(12)60560-1

166. Prescott HC, Osterholzer JJ, Langa KM, Angus DC, Iwashyna TJ. Late mortality after sepsis: propensity matched cohort study. BMJ (2016) 353:i2375. doi: 10.1136/bmj.i2375

167. Ou SM, Chu H, Chao PW, Lee YJ, Kuo SC, Chen TJ, et al. Long-term mortality and major adverse cardiovascular events in sepsis survivors. A nationwide population-based study. Am J Res Crit Care Med. (2016) 194:209-17. doi: 10.1164/rccm.201510-2023OC

168. Zorio V, Venet F, Delwarde B, Floccard B, Marcotte G, Textoris J, et al. Assessment of sepsis-induced immunosuppression at ICU discharge and 6 months after ICU discharge. Ann Intens Care (2017) 7:80. doi: 10.1186/s13613-017-0304-3

169. Makhoul IR, Sujov P, Smolkin T, Lusky A, Reichman B. Epidemiological, clinical, and microbiological characteristics of late-onset sepsis among very low birth weight infants in Israel: a national survey. Pediatrics (2002) 109:34-9. doi: 10.1542/peds.109.1.34
170. Lin CB, Hornik CP, Clark R, Cotten CM, Benjamin DK, Jr., CohenWolkoweiz M, et al. Very low birth weight neonates who survive early-onset sepsis do not have an increased risk of developing late-onset sepsis. Early Hum Dev. (2012) 88:905-9. doi: 10.1016/j.earlhumdev.2012.07.009

171. Wynn JL, Hansen NI, Das A, Cotten CM, Goldberg RN, Sanchez PJ, et al. Early sepsis does not increase the risk of late sepsis in very low birth weight neonates. J Pediatr. (2013) 162:942 -948.e3 doi: 10.1016/j.jpeds.2012.11.027

172. Miller JE, Hammond GC, Strunk T, Moore HC, Leonard H, Carter KW, et al. Association of gestational age and growth measures at birth with infectionrelated admissions to hospital throughout childhood: a population-based, data-linkage study from Western Australia. Lancet Infect. Dis. (2016) 16:95261. doi: 10.1016/S1473-3099(16)00150-X

Conflict of Interest Statement: The authors declare that the research was conducted in the absence of any commercial or financial relationships that could be construed as a potential conflict of interest.

Copyright (c) 2018 Hibbert, Currie and Strunk. This is an open-access article distributed under the terms of the Creative Commons Attribution License (CC BY). The use, distribution or reproduction in other forums is permitted, provided the original author(s) and the copyright owner(s) are credited and that the original publication in this journal is cited, in accordance with accepted academic practice. No use, distribution or reproduction is permitted which does not comply with these terms. 